\title{
Metal absorption lines as probes of the intergalactic medium prior to the reionization epoch
}

\section{Citation}

Furlanetto, Steven R., and Abraham Loeb. 2003. "Metal Absorption Lines as Probes of the Intergalactic Medium Prior to the Reionization Epoch." The Astrophysical Journal 588 (1): 18-34. https://doi.org/10.1086/374045.

\section{Permanent link}

http://nrs.harvard.edu/urn-3:HUL.InstRepos:41393348

\section{Terms of Use}

This article was downloaded from Harvard University's DASH repository, and is made available under the terms and conditions applicable to Other Posted Material, as set forth at http:// nrs.harvard.edu/urn-3:HUL.InstRepos:dash.current.terms-of-use\#LAA

\section{Share Your Story}

The Harvard community has made this article openly available. Please share how this access benefits you. Submit a story.

Accessibility 
ApJ, SubmitTed

Preprint typeset using LATEX style emulateapj v. 11/12/01

\title{
METAL ABSORPTION LINES AS PROBES OF THE INTERGALACTIC MEDIUM PRIOR TO THE REIONIZATION EPOCH
}

\author{
Steven R. Furlanetto ${ }^{1}$ \& Abraham Loeb ${ }^{1,2,3}$ \\ sfurlanetto@cfa.harvard.edu, loeb@ias.edu \\ ApJ, Submitted
}

\begin{abstract}
Winds from star-forming galaxies provide the most promising explanation for the enrichment of the intergalactic medium with heavy elements. Theoretical and observational arguments indicate that the pollution may have occurred at $z \gtrsim 6$; however, direct observational tests of such a scenario are needed. We model starburst winds in the high-redshift universe and find that the fraction of space filled by enriched material varies strongly with the assumed star formation efficiency $f_{*}$ and the fraction of supernova energy powering each wind, $f_{\text {esc }}$. We show that metals carried by these winds can be seen in absorption against bright background sources, such as quasars or gamma-ray bursts, in narrow lines with characteristic equivalent widths $\sim 0.5 \lesssim W \lesssim 5 \AA$. We argue that a substantial fraction of the metals in high-redshift winds are likely to reside in low ionization states (C II, O I, Si II, and Fe II), but higher ionization states (C IV and Si IV) could also provide useful probes of the winds. The number of such lines can constrain both $f_{*}$ and $f_{\text {esc }}$. Statistics of metal absorption lines can also be used to identify whether $\mathrm{H}_{2}$ is an efficient coolant in the early universe and to study the initial mass function of stars at high redshifts.
\end{abstract}

Subject headings: cosmology: theory - intergalactic medium-galaxies: high-redshift

\section{INTRODUCTION}

It is now obvious that mechanical feedback from galaxies and quasars has disturbed a significant fraction of the intergalactic medium (IGM). Types of feedback include relativistic quasar jets, winds from quasars, and winds from star-forming galaxies. These mechanisms have had a variety of consequences, including IGM heating (e.g., Voit et al. 2002), the suppression of star formation in the host galaxy (e.g., Springel \& Hernquist 2002) and in nearby galaxies (e.g., Scannapieco, Thacker, \& Davis 2001), and the generation of intergalactic magnetic fields (Kronberg, Lesch, \& Hopp 1999; Furlanetto \& Loeb 2001). Perhaps most importantly, feedback is responsible for enriching the IGM with heavy elements. Observations of the Ly $\alpha$ forest at $z \sim 3$ show that absorption systems with column densities $N_{\mathrm{HI}} \gtrsim 10^{14.5} \mathrm{~cm}^{-2}$ (corresponding to overdensities $\delta \gtrsim 5$; Schaye 2001) have metallicities $Z \sim 10^{-3}-10^{-2} Z_{\odot}$ (Tytler et al. 1995; Cowie et al. 1995; Songaila \& Cowie 1996; Ellison et al. 2000). Absorbers with $N_{\mathrm{HI}} \lesssim 10^{14.5} \mathrm{~cm}^{-2}$ require stacking or pixel-by-pixel analysis because of the weakness of the corresponding metal features, but recent studies suggest similar metallicities down to systems near the mean density of the IGM (Cowie \& Songaila 1998; Ellison et al. 1999, 2000; Aguirre, Schaye, \& Theuns 2002). These observations require that $\gtrsim 5 \%$ of space be enriched with heavy elements by $z \sim 3$ (Madau, Ferrara, \& Rees 2001). The era at which this pollution occurred is uncertain. Songaila (2001) found no evidence for evolution in the total mass-weighted abundance of C IV and Si IV between $z \sim 1.5-5$ (see also Qian et al. 2002). However, such mass-weighted measurements constrain only the metals in the most massive systems and tell us little about the total volume of enriched space. Furthermore, conclusions about the total metallicity based on single ionization states are fraught with uncertainty.

The history of metal enrichment has several important consequences for structure formation. Most directly, its extent mea- sures the volume over which galaxies have influenced their surroundings hydrodynamically, which in turn defines their feedback on the Ly $\alpha$ forest. Additionally, an early phase of metal injection may qualitatively change the characteristic mass of star formation (Bromm et al. 2001; Schneider et al. 2002; Mackey, Bromm, \& Hernquist 2002). The transition between zerometallicity star formation and "normal" star formation has important implications, e.g., for the expected redshift distribution of gamma-ray bursts (GRBs; Bromm \& Loeb 2002), for reionization (Wyithe \& Loeb 2002d; Cen 2002), and for the chemical abundance patterns of low-metallicity stars (Qian \& Wasserburg 2001, 2002).

Stars produced metals inside galaxies, and one must invoke some type of outflow to transport them throughout the IGM. Simple dynamical removal cannot pollute the large regions we observe (Aguirre et al. 2001c; but see Gnedin 1998). Radiation pressure-driven dust outflows may suffice (Aguirre et al. 2001a), although their effectiveness depends on many untested assumptions about the outflows, such as the geometry of the magnetic field. Quasar winds are unlikely by themselves to entrain enough metals to account for the observed metallicities, although they can help to disperse the metals over large volumes. Winds from star-forming galaxies are therefore the most likely mechanism. Correlated supernovae in starbursts can power "superwinds" with velocities $\sim 100-1000 \mathrm{~km} \mathrm{~s}^{-1}$ that reach far outside the host galaxy. Such winds have been studied in many local starburst galaxies (Lehnert \& Heckman 1996; Martin 1999) as well as in Lyman-break galaxies (LBGs) at $z \sim 3$ (Pettini et al. 2001, 2002; Shapley et al. 2002). Because their velocities exceed the escape speed from many of the hosts, we expect a subset of these outflows to penetrate into the general IGM. Escape is most likely from dwarf galaxies, because the amount of energy available to power a wind is proportional to the halo mass $M_{h}$ while the gravitational potential confining the wind increases with $M_{h}^{2}$. Thus, starburst winds can naturally

\footnotetext{
${ }^{1}$ Harvard-Smithsonian Center for Astrophysics, 60 Garden St., Cambridge, MA 02138

${ }^{2}$ Institute for Advanced Study, Princeton, NJ 08540

${ }^{3}$ Guggenheim Fellow
} 
account for a high-redshift episode of metal enrichment, when the characteristic mass of galaxies is small.

A great deal of theoretical work has gone into studying the galactic wind pollution mechanism. Ideally, the enrichment process should be modeled with cosmological simulations. To this end, several numerical simulations have incorporated galactic winds (e.g., Scannapieco, Thacker, \& Davis 2001; Springel \& Hernquist 2002; Theuns et al. 2002). Unfortunately, we do not yet fully understand the processes that generate winds even in nearby, well-observed starburst galaxies, although simulations are improving (Mac Low \& Ferrara 1999; Mori, Ferrara, \& Madau 2002). We must therefore insert simple parameterizations of the wind into the simulation. In order to understand the significance of this choice, most work to date has proceeded using analytic or semi-analytic methods (Nath \& Trentham 1997; Ferrara, Pettini, \& Shchekinov 2000; Madau, Ferrara, \& Rees 2001; Scannapieco, Ferrara, \& Madau 2002). Such models have the advantage of allowing much more flexible parameter studies. Because the relevant parameters are poorly constrained at present, the IGM filling factor (i.e., the fraction of space filled by enriched material) estimated by these studies varies widely (between $\sim 10^{-4}$ and $\sim 0.5$ ). Aguirre et al. (2001c) made a useful compromise between these two approaches by inserting winds into already completed cosmological simulations (see also Aguirre et al. 2001a,b). This allowed a broader parameter study than complete simulations and retained many of their advantages (such as the clustering of sources), while sacrificing a detailed treatment of the dynamical effects of the winds.

The crucial unknown parameters in galactic wind models fall into three broad categories. First, the characteristic redshifts and masses of the wind hosts are critical for setting up simulations with finite resolution and box size. Second, the unknown star formation efficiency at high redshifts has dramatic effects on both the sizes of individual wind bubbles (because it determines the energy input into the winds) and on the total amount of metals produced. Finally, a variety of questions about the structure of the winds remain unsettled. We do not know what fraction of the supernova energy is lost to radiation before entering the wind, what fraction of the galaxy mass is entrained by the wind, or the geometry of the winds. Studies of nearby starbursts can help to answer these questions; however, winds at high redshifts may have dramatically different characteristics. For example, local superwinds are bipolar, with the wind flowing perpendicular to the galaxy disk. If star formation precedes disk formation in the halo, we would expect high-redshift outflows to be more isotropic (Madau, Ferrara, \& Rees 2001).

Ultimately, we can only answer these questions through observations of the pollution mechanism. One promising technique is to study absorption lines caused by metals in the winds. In both local starbursts (Phillips 1993; Lequeux et al. 1995; Heckman \& Leitherer 1997; Heckman et al. 2000) and LBGs (Pettini et al. 2001, 2002; Shapley et al. 2002) absorption features can be observed through moderate resolution spectroscopy of the host galaxy. Unfortunately, the hosts of highredshift $(z \gtrsim 6)$ starbursts will be too faint to serve as useful background lights. We therefore must use other bright background sources, such as quasars and GRBs, against which absorption can be seen. Such background sources have the distinct advantage of allowing us to probe "relic" winds whose host galaxies no longer actively form stars. Relic wind bubbles are older and larger than the young winds in starburst galax- ies; they are also more common than active winds because only a fraction of galaxies host starbursts at any time. Thus relic bubbles fill more space than active winds, and a careful study of their properties is crucial. Similar observations have been attempted for local starbursts (Norman et al. 1996) and an extension of this technique has been used to study the effects of LBGs on the IGM (Adelberger et al. 2002). Oh (2002) has also proposed using metal absorption lines to study the reionization process, provided that metals have been injected relatively uniformly in dense regions by $z \sim 6$.

The neutral fraction of IGM hydrogen is relatively large ( $\gtrsim 1 \%$ ) before the completion of reionization at $z_{r} \gtrsim 6$ (Becker et al. 2001; Fan et al. 2002). The resulting absorption blueward of the Ly $\alpha$ transition (Gunn \& Peterson 1965) will render unobservable any metal lines with rest-frame wavelength $\lambda_{m}$ in the range $\left(1+z_{r}\right) /(1+z)<\lambda_{m} / \lambda_{\text {Ly } \alpha}<1$, where $\lambda_{\text {Ly } \alpha}=1216 \AA$ and $z$ is the redshift of the absorbing system. A transition below the left-hand limit will be observable provided that it can be separated from the Ly $\alpha$ forest. A transition with $\lambda_{m}>\lambda_{\mathrm{Ly} \alpha}$ is unaffected by neutral hydrogen inside a finite redshift interval $z_{\min }<z<z_{s}$, where $z_{s}$ is the redshift of the background source and

$$
1+z_{\min }=\left(1+z_{s}\right) \frac{\lambda_{\mathrm{Ly} \alpha}}{\lambda_{m}} .
$$

Within this range, metal absorption lines will remain visible. A collection of sources with a range of $z_{s}$ should therefore allow us to probe winds throughout the high- $z$ IGM.

In this paper, we use a simple semi-analytic model for winds from star-forming galaxies to study how observations of metal absorption by wind bubbles in the era before reionization can constrain the metal enrichment process. We compute the halo star formation histories and follow the expansion of their winds into the IGM. We show that metals carried by the winds produce observable absorption features when seen against bright background sources, and that with current and future instruments, observations of these lines can constrain the star formation efficiency in the early universe and can distinguish between different halo cooling scenarios and stellar initial mass functions.

We discuss our supernova wind model in $\$ 2$ and metals in the high-redshift IGM in $\S 3$. We present our predictions for the statistics of metal absorbers in $\$ 4$ and discuss our results in $\S 5$. We assume the standard $\Lambda$ CDM cosmological parameters of $\Omega_{m}=0.3, \Omega_{\Lambda}=0.7, \Omega_{b}=0.05, \sigma_{8}=0.9, n=1$, and a Hubble constant $H_{0}=100 h \mathrm{~km} \mathrm{~s}^{-1} \mathrm{Mpc}^{-1}$. We set $h=0.7$ in our numerical calculations.

\section{SUPERNOVA WIND MODEL}

In this section we describe our model for superwinds in detail. We discuss the abundance of wind sources and their star formation histories in $\$ 2.1$. We fix the mechanical power driving the winds in $\S 2.2$, and we describe our model for their expansion into the IGM in $\S 2.3$.

\subsection{Halo Abundances and Star Formation Histories}

At any redshift, the halo mass function $d n / d M$ determines the number of wind sources: each star-forming halo has the potential to drive such a wind. We use the Press \& Schechter (1974) formalism to calculate the mass function, with the modifications suggested by Sheth \& Tormen (1999) to better match numerical simulations (Jenkins et al. 2001).

A halo can only form stars if its mass exceeds a threshold

$$
M_{\min }(z)=\max \left[M_{\mathrm{fil}}(z), M_{\mathrm{cool}}(z)\right] \text {. }
$$


The first condition requires that the halo mass exceeds the "filter mass" $M_{\text {fil }}$, or the effective Jeans mass in the evolving IGM (Gnedin \& Hui 1998). The second condition requires halo gas to cool efficiently after virialization. In the low metallicity halos we study, line transitions of atomic and molecular hydrogen are the only effective coolants. Atomic cooling is efficient in halos with $T_{\text {vir }} \gtrsim 10^{4} \mathrm{~K}$, where the virial temperature $T_{\text {vir }}$ is defined in equation (26) of Barkana \& Loeb (2001). In most of our work, we will fix $M_{\text {cool }}$ by requiring that atomic cooling can proceed. The vibrational lines of $\mathrm{H}_{2}$ make it an efficient coolant in halos with $T_{\text {vir }} \gtrsim 400 \mathrm{~K}$. It is currently unknown when (and even whether) $\mathrm{H}_{2}$ is destroyed by photons below the ionization threshold of atomic hydrogen (Haiman, Rees, \& Loeb 1997; Haiman, Abel, \& Rees 2000), or whether radiative feedback from early generations of stars enhances the formation of $\mathrm{H}_{2}$ (Ricotti, Gnedin, \& Shull 2002). We therefore also show results in which $\mathrm{H}_{2}$ cooling is permitted.

The power driving a wind is ultimately determined by the star formation history of the host halo, which is usually associated with the halo merger history. We use a Monte Carlo implementation of the extended Press-Schechter formalism (Lacey $\&$ Cole 1993) to choose histories for each halo. Given a halo of mass $M_{h}$ at redshift $z_{f}$, this algorithm determines the probability distribution of progenitor masses $\left(M_{1}, M_{h}-M_{1}\right)$ that merged to form the object in a small redshift interval $d z$ around $z_{f}$ (we choose $d z$ such that the probability of a major merger is $<1 \%$ ). For each halo, we construct the merger history by first choosing $M_{1}$ from this distribution. We then choose the larger of the two progenitor masses (i.e., $M_{1}$ if $M_{1}>M_{h} / 2$ and $M_{h}-M_{1}$ otherwise) and repeat the process about a redshift $z_{f}+d z$. We continue this procedure until the progenitor mass is smaller than $M_{\text {min }}$ at a redshift $z_{\text {form }}$.

In order to simplify our calculations, we divide the merger history into discrete events in which the halo mass has grown by $>50 \%$. (We find that our results are insensitive to the value of this threshold.) We assume that in each such event a fraction $f_{*}$ of the accreted baryonic mass $M_{g}$ is transformed into stars on a timescale $t_{\text {sf }}$. We assume that each merger occurs over the free-fall time of the host halo $t_{\mathrm{ff}}$, and we normally set $t_{\mathrm{sf}}=t_{\mathrm{ff}}$. In the rare occasions in which another merger event begins within $t_{\mathrm{ff}}$ of the first, we set $t_{\text {sf }}$ equal to the time between these events. In fact, our results are relatively insensitive to $t_{\mathrm{sf}}$. Although the wind dynamics do change during the "active" phase of the wind, most of the expansion occurs after active star formation has ended as long as $t_{\mathrm{sf}} \ll H(z)^{-1}$. If this condition is satisfied, the resulting bubble sizes do not depend strongly on $t_{\mathrm{sf}}$ because bubbles spend most of their time in the late "remnant" stages. However, if $t_{\mathrm{sf}} \sim H(z)^{-1}$, a significant amount of energy is lost to radiative cooling. In this case the total volume filled by metal-enriched winds can decrease by $\sim 25 \%$.

With this prescription, we implicitly assume that a progenitor does not form stars and release mechanical energy until it joins the "trunk" of the merger tree. In reality, as long as the progenitor mass exceeds $M_{\min }(z)$, it would have formed stars before merging and thus be surrounded by its own wind bubble. The two bubbles will presumably merge along with the galaxies in some complicated manner. Our prescription is reasonable so long as the total wind energy is approximately conserved during the merging process. Note also that by choosing to follow only one of the progenitors at each branching, we do not necessarily track the largest single progenitor existing at each step. This error is not important so long as our Monte Carlo prescrip- tion includes a sufficiently large number of trials.

\subsection{Wind Luminosity}

Once we have fixed the rate of star formation $f_{*} M_{g} / t_{\mathrm{sf}}$, the mechanical luminosity in the wind depends only on the initial mass function (IMF) of the stars and the fraction of supernova energy available to power the wind. The IMF dependence can be characterized entirely by the total amount of stellar mass that must be formed in order to produce one supernova, $\omega_{\mathrm{SN}}$. For a Scalo (1998) IMF with lower and upper mass cutoffs $M_{l}=0.1 \mathrm{M}_{\odot}$ and $M_{u}=100 \mathrm{M}_{\odot}, \omega_{\mathrm{SN}}=126 \mathrm{M}_{\odot}$, while for a Salpeter (1955) IMF with the same mass cutoffs, $\omega_{\mathrm{SN}}=135 \mathrm{M}_{\odot}$. We use a Scalo IMF in the following because it better matches observations of the IMF in the local universe; however, the results are clearly insensitive to this choice.

Recent simulations of the formation of metal-free stars indicate that these objects may be very different from nearby stars, with typical masses $\sim 10^{2}-10^{3} \mathrm{M}_{\odot}$ (Abel, Bryan, \& Norman 2002; Bromm, Coppi, \& Larson 2002). This mass scale is determined by the physics of $\mathrm{H}_{2}$ cooling; we therefore assume that when molecular hydrogen is an active coolant, star formation proceeds with a Very Massive Star (VMS) IMF. Unfortunately, these simulations do not yet have the dynamic range to follow the collapse to nuclear burning, so they are unable to predict the final IMF or the allowed mass range of metal-free stars. We therefore assume (somewhat arbitrarily) that the IMF is a Salpeter-like power law with an index $\beta=2.35$ and mass cutoffs $M_{l}=100 \mathrm{M}_{\odot}$ and $M_{u}=500 \mathrm{M}_{\odot}$. Interestingly, massive, low-metallicity stars leave very different remnants from "normal" stars (Heger \& Woosley 2002). For a stellar mass less than $130 \mathrm{M}_{\odot}$ or greater than $260 \mathrm{M}_{\odot}$, the star collapses to a black hole without significant matter or energy ejection. However, stars with masses $130 \mathrm{M}_{\odot} \lesssim M \lesssim 260 \mathrm{M}_{\odot}$ are subject to the $e^{+} e^{-}$pair instability and explode without leaving a remnant black hole. Unlike normal supernovae, such explosions expel the highly-enriched core along with the envelope. This mechanism may thus have significant consequences for metal enrichment (see $\$ 3.1$ below). For our VMS IMF, we find $\omega_{\mathrm{SN}}=462 \mathrm{M}_{\odot}$.

We assume that each supernova releases an energy $E_{\mathrm{SN}}=$ $10^{51} E_{51}$ erg into the interstellar medium of the host galaxy. For a Scalo IMF, we assume $E_{51}=1$. For a VMS IMF, we assume $E_{51}=10$, an approximate logarithmic average of the mass-dependent explosion energy in the models of Heger \& Woosley (2002). However, only a fraction $f_{\text {esc }}$ of this energy is available to power the wind: the rest is lost, primarily to radiative cooling, in the early stages of the outflow. Observations of the cool, dense ejecta of starbursts in the local universe require $f_{\text {esc }} \gtrsim 0.1$ (Heckman et al. 2000), while observations of the warm and hot plasma in the wind imply $f_{\text {esc }} \gtrsim 0.3$ (Strickland et al. 2000). However, at high redshifts the characteristic density is much higher, and radiative cooling could in principle be more important. Simulations of high-redshift starbursts suggest that $f_{\text {esc }} \sim 0.25$ (Mori, Ferrara, \& Madau 2002).

With these parameters, the mechanical luminosity driving the wind is

$$
L_{w}=10^{39}\left(\frac{f_{*}}{0.1} \frac{f_{\mathrm{esc}}}{0.25} E_{51} \frac{126 \mathrm{M}_{\odot}}{\omega_{\mathrm{SN}}} \frac{10^{8} \mathrm{yr}}{t_{\mathrm{sf}}} \frac{M_{g}}{10^{7} \mathrm{M}_{\odot}}\right) \mathrm{erg} \mathrm{s}^{-1} .
$$

\subsection{Wind Model}


We can now calculate the expansion of the wind into the IGM. The wind begins when the progenitor mass first exceeds $M_{\text {min }}(z)$, and energy is added to it during each merger event. We use the thin-shell approximation (Tegmark, Silk, \& Evrard 1993; Furlanetto \& Loeb 2001), which is well-justified in a cosmological context (Ikeuchi, Tomisaka, \& Ostriker 1983). The expanding wind sweeps a fraction $1-f_{m}$ of the ambient IGM into a thin shell. The remaining gas leaks into the hot, rarefied interior whose pressure drives the shell expansion. Assuming spherical symmetry, we can then describe the outflow through the following system of equations:

$$
\begin{aligned}
\ddot{R}= & \frac{4 \pi R^{2}}{M_{s}}\left(p-p_{\mathrm{ext}}\right)-\frac{G}{R^{2}}\left(M_{d}+M_{\mathrm{gal}}+\frac{M_{s}}{2}\right) \\
& +\Omega_{\Lambda}(z) H^{2}(z) R-\frac{\dot{M}_{s}}{M_{s}}\left(\dot{R}-v_{\mathrm{inf}}\right), \\
\dot{p}= & \frac{L}{2 \pi R^{3}}-5 p \frac{\dot{R}}{R}, \\
\dot{M}_{s}= & \begin{cases}0, & v_{\text {inf }} \geq \dot{R}, \\
4 \pi R^{2} \rho_{g}\left(\dot{R}-v_{\text {inf }}\right), & v_{\text {inf }}<\dot{R} .\end{cases}
\end{aligned}
$$

Here, $R$ is the shell radius in physical units, $M_{s}$ is the shell mass, and $p$ is the pressure of the hot bubble interior. The shell expands through the host halo and into the IGM; the ambient baryon density, infall velocity field, and external pressure are denoted $\rho_{g}, v_{\text {inf }}$, and $p_{\text {ext }}$, respectively. We assume that the host galaxy has photoionized the external medium, which is likely to be true during most of the wind's expansion. The enclosed dark matter mass is $M_{d}$ and the host galaxy mass (which can be time dependent as the wind entrains material from the host) is $M_{\mathrm{gal}}$. We use the universal halo profile of Navarro, Frenk, \& White (1997) inside the accretion shock radius and the selfsimilar solution of Bertschinger (1985) outside of this radius, although the details of the profile are not critical to our results. Each halo is therefore embedded in a relatively small overdense infall region, which is in turn surrounded by IGM at the mean cosmic density. Both $M_{h}$ and the halo profile evolve as the host galaxy grows through accretion. Finally, the rate of energy input $L$ into the bubble interior is

$$
L=L_{w} \Theta\left(t-t_{\mathrm{sf}}\right)+L_{\mathrm{diss}}-L_{\mathrm{comp}},
$$

where $L_{\text {comp }}$ is the inverse Compton cooling luminosity, $L_{w}$ is the wind luminosity defined in equation $(3), \Theta$ is the Heaviside step function, and

$$
L_{\mathrm{diss}}=-\frac{1}{2} f_{d} \dot{M}\left(\dot{R}-v_{\mathrm{inf}}\right)^{2} .
$$

Here $f_{d}$ is the fraction of the energy dissipated by the acceleration of ambient material to the shell velocity that is retained by the outflow (rather than lost through cooling inside the shell). In the hot $\left(T \sim 10^{6} \mathrm{~K}\right)$, rarefied bubble cavity, inverse Compton cooling dominates at the high redshifts we consider, with a cooling time

$$
\frac{t_{\text {comp }}}{H(z)^{-1}} \approx 0.4\left(\frac{10}{1+z}\right)^{5 / 2} .
$$

We therefore neglect metal line and free-free cooling inside the bubble. ${ }^{4}$ Material in the shell will cool rapidly through either inverse Compton cooling (when it is ionized) or line cooling (once recombination occurs). We therefore set $f_{d}=0$, although we also show some results for $f_{d}=1$. We describe the expansion model in more detail in Furlanetto \& Loeb (2001).

As emphasized above, the structure of high-redshift winds is not well-constrained. They are likely to be more isotropic than those of local starbursts because their disks are not as welldefined. LBGs appear to host isotropic outflows, because wind absorption lines are seen in all LBGs that have been observed (Shapley et al. 2002). Moreover, the outflow will likely become more spherical as it plows into the IGM. We therefore assume that all winds expand spherically. A second key question is whether star formation occurs before or after the halo gas collapses into a dense medium. In the former case, the wind expands into a spherical, relatively low density medium (see, e.g., Madau, Ferrara, \& Rees 2001). In this "no disk formation" model, we assume that supernovae inject energy into the center of the halo and that all of the halo gas is swept up by the shell. Simulations show that a substantial fraction $(\sim 0.5)$ of the gas in the halo may remain inside the protogalaxy (Mori, Ferrara, \& Madau 2002); however, this choice makes only a small difference to our results because most of the expansion occurs after the swept-up IGM mass exceeds the halo mass (see §4.3).

In most of our work, we assume that the halo gas collapses to a dense medium before the starburst begins, leaving the rest of the halo empty of baryons. We assume that the dense medium has a scale length

$$
R_{d}=\frac{\lambda}{\sqrt{2}} R_{\mathrm{vir}}
$$

where $R_{\text {vir }}$ is the virial radius of the halo as defined in equation (24) of Barkana \& Loeb (2001) and $\lambda=0.05$ is the spin parameter (Mo, Mao, \& White 1998). With a slight abuse of terminology, we refer to this case as the "disk formation" scenario. Note that we do not require well-defined disks to form, only that the gas has collapsed to a dense central object. We assume that the wind entrains a fraction $f_{\mathrm{sw}}$ of the protogalactic material. Motivated by observations of local disk starbursts, we set $f_{\text {sw }}=2 f_{*}$ in most of what follows (Martin 1999). ${ }^{5}$ The initial velocity of the outflow $v_{w}$ is then fixed (c.f., Aguirre et al. 2001c; Springel \& Hernquist 2002):

$$
v_{w}=310\left(\frac{f_{\mathrm{esc}}}{0.25} \frac{2}{f_{\mathrm{sw}} / f_{*}} \frac{126 \mathrm{M}_{\odot}}{\omega_{\mathrm{SN}}}\right)^{1 / 2} \mathrm{~km} \mathrm{~s}^{-1}
$$

Note that $v_{w}$ is independent of halo mass, implying that winds will most easily escape the gravitational attraction of smaller halos. The wind velocities in nearby starbursts are inferred to be independent of the host mass (Martin 1999).

Our prescription for the initial conditions depends on whether disk formation is allowed. If it is not, we use the selfsimilar wind-fed solution of Ostriker \& McKee (1988) to calculate the time for the wind to travel to $R_{d}$ assuming a constant density within this region. (Although $R_{d}$ has no physical meaning in this case, we use it to fix numbers.) The self-similar solution also yields the initial velocity, and we assume that the remaining supernova energy goes into the bubble pressure. While

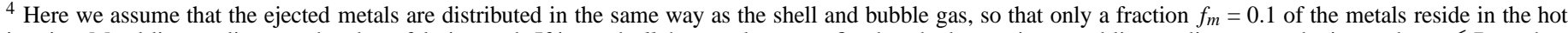

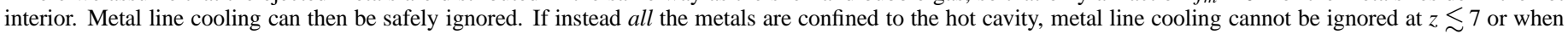

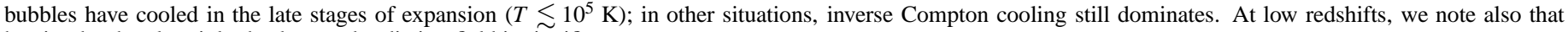
heating by the ultraviolet background radiation field is significant.

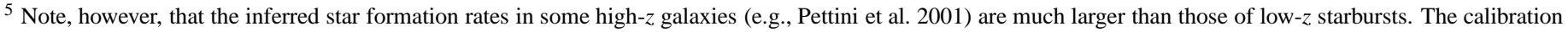
of Martin (1999) may therefore not apply to these systems. Fortunately, our results are relatively insensitive to this choice (see Figure 11).
} 
this prescription makes several simplifying assumptions, the results are insensitive to the precise initial conditions in this case. If disk formation is allowed, we assume that the wind travels with a constant velocity $v_{w}$ within the dense collapsed region. The initial time is determined by the time taken for the wind to reach $R_{d}$. For numerical stability, we convert half of the kinetic energy to the thermal energy of the bubble interior before beginning the integration. (The final result is not sensitive to this conversion.)

When gravity, density variations, external pressure, radiative cooling, and the cosmological expansion are ignored, our model for a single explosion recovers the usual Sedov-Taylor solution if $f_{d}=1$ or the pressure-driven snowplow solution if $f_{d}=0$ (Ostriker \& McKee 1988). With these effects included, we find instead $R \propto E^{0.35}$ at fixed halo mass, a somewhat steeper energy dependence than expected in self-similar solutions. This is principally due to the deceleration of the Hubble flow: an explosion with smaller energy takes longer to escape the host halo and therefore must expend more energy accelerating the sweptup IGM. Our model matches well with previous analytic work on outflows (e.g., Barkana \& Loeb 2001). However, it predicts wind radii approximately twice those found by Madau, Ferrara, $\&$ Rees (2001), even when we include only the effects that are part of their model. We do not consider this a cause for concern, because the simulations of Mori, Ferrara, \& Madau (2002) have a similar discrepancy and compare favorably with our results.

Figures 1 and 2 show the physical sizes $R$ and expansion velocities $v_{\exp }$ of supernova wind bubbles at $z=20,12,8$, and 3 as a function of host halo mass. We assume the following parameters: $f_{*}=0.1, f_{\mathrm{esc}}=0.25, \omega_{\mathrm{SN}}=126 \mathrm{M}_{\odot}$ (corresponding to a Scalo IMF), atomic cooling, disk formation, and $f_{d}=0$. The lower mass limit in each panel is simply $M_{\min }$, and the upper limit is the approximate mass $M_{\max }$ above which the winds cannot escape the host halo. At a given mass, the dispersion in radius and velocity is caused by the randomly chosen star formation histories. For halos near $M_{\min }, R$ varies significantly because such halos have undergone only a few star formation episodes. Thus, the time elapsed since the wind began fluctuates strongly. As $M_{h}$ increases, the number of mergers increases and the dispersion decreases. Recall that the wind begins when the progenitor crosses the cooling threshold, and each subsequent merger adds energy to it. Because massive halos have undergone many such random merger events, the fluctuations in merger times average out and the scatter in $R$ is fairly small. The dispersion increases again near $M_{\max }$, where the star formation history, detailed wind model, and initial conditions all have a substantial effect on whether the wind can escape beyond $R_{\mathrm{vir}}$. Fortunately, halos near $M_{\max }$ are sufficiently rare that they have negligible effects on our final results.

$R$ increases rapidly as redshift decreases because of the increasing cosmic time available to the outflow. The dispersion also increases with redshift, because small variations in the formation time make larger differences when the total lifetime of the outflow is small. The weak dependence of radius on mass is primarily due to the gravitational binding of the host. Because the energy available to the starburst scales with $M_{h}$ while the gravitational potential scales with $M_{h}^{2}$, we expect $R\left(M_{h}\right)$ to flatten as mass increases until eventually the winds can no longer escape outside the virial radii of the hosts (shown by the solid curves). For most of the redshift range of interest and for reasonable choices of parameters, the threshold halo mass is $M_{\max } \sim 10^{10}-3 \times 10^{11} \mathrm{M}_{\odot}$, increasing as $z$ decreases be- cause halos become less concentrated. Well below this threshold mass, we find $R \propto M_{h}^{\sim 1 / 5}$, as would have been expected in a naive Sedov-Taylor solution (though the agreement is coincidental and the scaling only approximate). The top left panel of Figure 1 shows that LBGs can directly affect the surrounding IGM to (physical) distances $\sim 100 \mathrm{kpc}$, provided that they reside in halos with $M_{h} \sim 10^{11} \mathrm{M}_{\odot}$ (see also Aguirre et al. 2001b). Interestingly, Adelberger et al. (2002) find that regions within $\sim 125 \mathrm{kpc}$ of LBGs have unexpectedly small amounts of $\mathrm{H}$ I. One possible explanation is that winds have cleared this region of $\mathrm{H} \mathrm{I}$; if so, these observations suggest that our model provides a reasonable description of the extent of outflows.

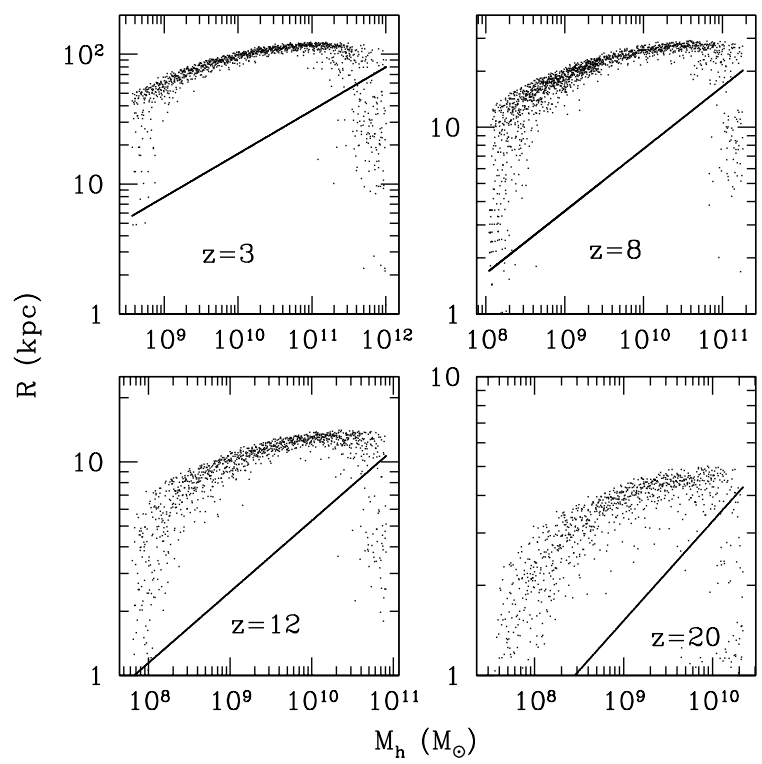

FIG. 1.- Physical radius $R$ of wind bubbles at various redshifts, as a function of halo mass. The solid line in each panel shows the virial radius $R_{\text {vir }}$ of the host halo. Note that both the mass and radius scales change between the different panels. All panels assume our standard set of parameters.

Figure 2 shows that all of our wind bubbles have characteristic expansion velocities $v_{\exp } \sim 50 \mathrm{~km} \mathrm{~s}^{-1}$, with a substantial dispersion. The velocity increases slowly with mass, particularly at low redshifts, until $M_{h} \sim M_{\max }$. Our expansion velocities are much smaller than those inferred from observed starburst winds (Heckman et al. 2000; Pettini et al. 2002). The difference is, however, easily understood. While existing observations probe the early stages of starburst winds, our models primarily sample the late time behavior. At early times, the material ejected from the host galaxy dominates the dynamics, but gravity and collisions with the ambient IGM inevitably decelerate the winds later on. We can estimate $v_{\exp }$ by assuming that the host experiences one major merger per Hubble time and that all of the energy released goes into accelerating the swept-up medium. The average expansion velocity is then

$$
\begin{array}{rl}
v_{\exp } \sim 50 & \mathrm{~km} \mathrm{~s}^{-1}\left(\frac{f_{*}}{0.1} \frac{f_{\mathrm{esc}}}{0.25} \frac{126 \mathrm{M}_{\odot}}{\omega_{\mathrm{SN}}} \frac{M_{h}}{10^{9} \mathrm{M}_{\odot}}\right)^{1 / 3} \\
& \times\left(\frac{20 \mathrm{kpc}}{R}\right)^{2 / 3}\left(\frac{10}{1+z}\right)^{1 / 2} .
\end{array}
$$

This simple estimate neglects cooling, gravity, and the Hubble flow energy contained in the IGM, but it illustrates the approximate velocities we find in our model. Recalling $R \propto M_{h}^{1 / 5}$ for $M_{h} \ll M_{\text {max }}$, equation (12) also predicts a weak mass depen- 
dence $v_{\exp } \propto M_{h}^{1 / 5}$, near what we observe in the complete model at small redshifts. Note that the Mach number $\mathcal{M} \equiv v_{\exp } / c_{s} \sim 4$, where $c_{s}$ is the sound speed of the photo-ionized IGM. The thin shell model assumes $\mathcal{M} \gg 1$, so it is only marginally valid in this regime. The detailed expansion may therefore differ somewhat from our model.

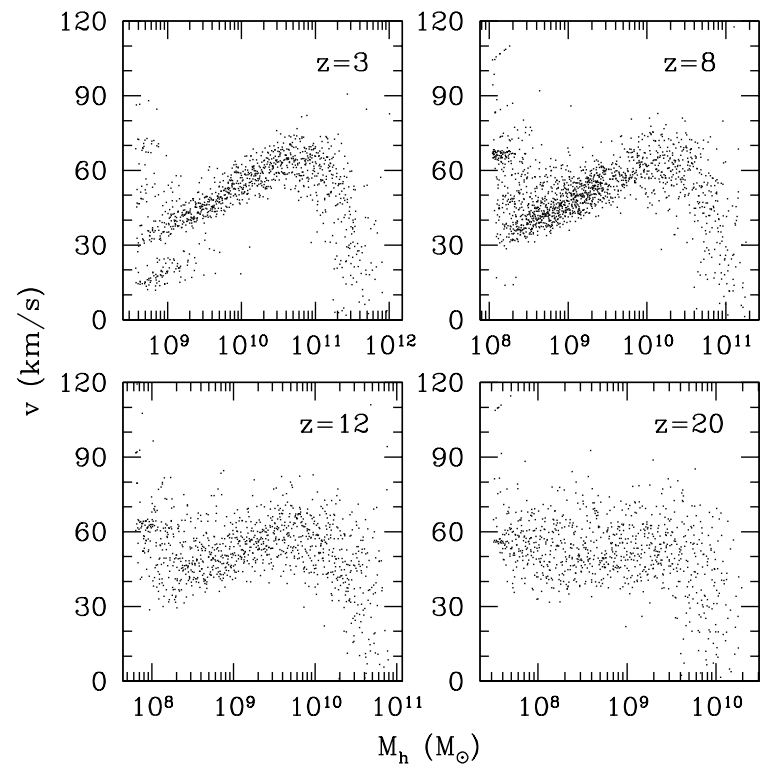

FIG. 2.- Expansion velocity $v_{\exp }$ of wind bubbles at various redshifts, as a function of halo mass. Note that the mass scale changes between the different panels. All panels assume our standard set of parameters.

\section{METAL LINES AT HIGH-REDSHIFT}

We now calculate the distribution of metals within the wind bubbles, including the most abundant elements and ionization states as well as the geometry of the metal-polluted regions.

\subsection{Metal Yields}

The relevant elements are easy to determine: they are those atoms most abundantly produced by Type II supernovae (for a Scalo IMF) or by pair instability supernovae (for a VMS IMF). We summarize the average yields per supernova $Y_{x}$ for these elements in Table 1. For Type II supernovae, we use the yields of Woosley \& Weaver (1995) averaged over a Scalo IMF with $Z=10^{-4} Z_{\odot}$. These yields depend on the energy of the supernova, which introduce uncertainties of $\sim 25 \%$. We have conservatively taken the yields from the lower energy model. The metallicity of the progenitor star introduces a similar uncertainty, but we do not include chemical evolution within galaxies because other simplifications in our model create uncertainties at least as large. For the VMS scenario, we average the yields of Heger \& Woosley (2002) over our power-law IMF.

Table 1 also lists the properties of several interesting transitions, including the wavelength $\lambda_{m}$ and the oscillator strength $f_{\text {osc }}$. We will focus on these lines in the following.

\subsection{Wind Structure}

Next we must determine how the ions are distributed throughout the wind. Note that we assume purely spherical expansion throughout. In reality, each halo is surrounded by the "cosmic web" of filaments and voids. Dense filaments will slow the shell and break spherical symmetry. Fortunately, they fill only a small fraction of space and will not strongly affect the total volume of the bubble (e.g., Theuns et al. 2002). A more serious problem is fragmentation (see below).

Observations of both local starburst winds (Heckman et al. 2000) and LBG outflows (Pettini et al. 2002) show very complex ionization structure, with absorption from both low ionization states typical of $\mathrm{H}$ I regions (such as C II, O I, Si II, and Fe II) and high-ionization states (such as C IV and Si IV). Within the wind region, the hot gas is inferred to have a very high covering fraction $C_{A}$ (defined as the fraction of the crosssectional area covered by the gas), while the low-ionization gas has $C_{A} \sim 0.4-0.8$ depending on the source (Heckman et al. 2000; Shapley et al. 2002).

If mixing between the ejected gas and the swept-up IGM is efficient, the thin-shell model requires that a fraction $1-f_{m}$ of the metals reside in a cool, dense shell with $T \sim 10^{4} \mathrm{~K}$, with the remainder in the hot, rarefied interior. Although there is good evidence for a shell structure in many observations of starburst winds (Martin 1998; Cecil et al. 2001), other observations suggest that the cool gas resides in clouds entrained by the wind (Heckman et al. 2000). Even though there is often little evidence for a cool shell in starbursts of the latter type, the related observations exclusively study young starbursts in which the wind mass is still dominated by gas ejected from the host galaxy. Once the swept-up IGM gas dominates the wind, a shell will inevitably form (thought it may not be spherical). It is not clear whether the metals will fully mix with the swept-up material that constitutes most of the shell mass. However, in our model the shell itself travels with $v_{\exp } \sim 50 \mathrm{~km} \mathrm{~s}^{-1}$ while winds are launched with $v_{w} \sim 300 \mathrm{~km} \mathrm{~s}^{-1}$ (see equation [11]). For $M \ll M_{\max }$, entrainment of the ambient IGM causes most of the deceleration; therefore metals in the wind will not decelerate significantly until they impact the shell at $t \sim 5 \times 10^{6}(R / \mathrm{kpc}) \mathrm{yr}$, where mixing through turbulence or diffusion can occur.

However, the shell may eventually fragment into clouds due to cooling, Rayleigh-Taylor instabilities, turbulence, or inhomogeneities in the ambient medium. Fragmentation is particularly likely given the relatively modest Mach number of the flow (see \$2.3). At $z=3$, an analysis similar to our own shows that galactic winds must fragment in order not to overproduce Ly $\alpha$ absorption lines (Theuns, Mo, \& Schaye 2001). If either the shell has fragmented or the metals are distributed in cold clouds throughout the bubble, only a fraction $C_{A}<1$ of all lines of sight through the wind bubble will produce metal absorption lines. On the other hand, each line of sight that does pass through a cloud will have on average a larger column density of metals. For unsaturated lines, the equivalent width $W \propto N_{x} \propto C_{A}^{-1}$, where $N_{x}$ is the column density of the relevant ion. In this regime (which is satisfied for most of the lines of interest; see §4.1), we then have the transformation

$$
\frac{d N(>W)}{d z} \rightarrow C_{A} \frac{d N\left(>C_{A} W\right)}{d z},
$$

where $d N(>W) / d z$ is the number of absorption systems intersected per unit redshift with equivalent width greater than $W$ along a random line of sight (see §4.3). For saturated lines, the equivalent width transformation factor will be smaller. Because our results can be scaled relatively easily with $C_{A}$, for concreteness we assume that $C_{A}=1$. We further discuss the consequences of fragmentation in $\S 5$.

The wind structure will also determine the width of the metal lines. In the shell (with $T \sim 10^{4} \mathrm{~K}$ ), the thermal line widths are $b_{\mathrm{th}} \sim 3.7\left(12 / A_{x}\right)^{1 / 2} \mathrm{~km} \mathrm{~s}^{-1}$, where $b$ is the Doppler parameter 
and $A_{x}$ is the atomic mass of the ion $x$. In this case, though the lines may be very deep, they will be extremely narrow and hence difficult to detect except with extremely high-resolution infrared spectroscopy. However, low and moderate resolution spectroscopy of both nearby starbursts and LBG outflows show line widths comparable to or greater than the inferred mean expansion velocity of the wind (Heckman et al. 2000; Pettini et al. 2002). This can be easily understood if cloud fragments, each with an intrinsic line width $b_{\text {th }}$, are distributed throughout the wind with a velocity dispersion comparable to $v_{\exp }$, or if clouds are continuously produced and accelerated by the wind. We assume in the following that $b=v_{\exp }$. Note that the expansion velocities we find $\left(b \sim 50 \mathrm{~km} \mathrm{~s}^{-1}\right.$; see $\left.\$ 2.3\right)$ are much smaller than those typically observed in young starburst winds. If star formation is ongoing, and if the fast wind near the host contains a substantial fraction of the metals, we will underestimate the true line width. However, as long as the lines are not saturated, the equivalent width $W$ is independent of our choice for $b$. Even if the lines are saturated, we cannot overestimate $W$ so long as we underestimate the velocity dispersion. We emphasize again that the observations from which we calibrate $b=v_{\exp }$ use the starburst galaxy itself as the background light source; thus, they only constrain the active phases of the starburst. The old winds that we study through background quasar spectra may have very different velocity dispersions.

\subsection{Ionization States}

Given the most abundant elements, we now determine the relevant ionization states. Three ionizing processes must be included: collisional ionization (especially in the hot interior), photoionization from the host galaxy, and photoionization by the extragalactic background light. In general, if a fraction $C_{m}$ of a given element is in a specified ionization state, the covering fraction of the outflow is unaffected but $N_{x} \propto C_{m}$. Thus in the unsaturated line limit

$$
\frac{d N(>W)}{d z} \rightarrow \frac{d N\left(>C_{m}^{-1} W\right)}{d z}
$$

$C_{m}<1$ can also occur if metals are retained by the host galaxy. However, simulations suggest that the wind carries a very large fraction of the metals produced during a starburst (Mac Low \& Ferrara 1999).

In the thin shell model, we can estimate the ionization state of the metals in each phase. We first consider metals in the shell. If the hydrogen in the shell is neutral, metals will reside in states typical of $\mathrm{H}$ I regions (C II, O I, Si II, and Fe II for the elements of interest), because photons able to further ionize these elements will be absorbed by $\mathrm{H}$ I. The ionization potential of $\mathrm{O} \mathrm{I}$ is $13.62 \mathrm{eV}$, and it remains locked in charge exchange equilibrium with H I (see Oh 2002 for a discussion of the utility of this mechanism). The other elements have first ionization potentials below that of hydrogen. We now show that the extragalactic background light most likely suffices to maintain these first ionized states. The total column density of a metal $x$ along a typical line of sight through (each side of) the wind is

$$
N_{x, \text { tot }} \approx \frac{Y_{x}}{\omega_{\mathrm{SN}}} \frac{\Omega_{b}}{\Omega_{0}} \frac{f_{*} M_{h}}{4 \pi A_{x} m_{p} R^{2}} .
$$

We can estimate the background flux $J\left(\nu_{\text {ion }}\right) \equiv J_{\text {ion }}$, where $\nu_{\text {ion }}$ is the ionization threshold of the relevant species, needed to keep a metal ionized by requiring that the ionization rate exceed the recombination rate within the shell: $J_{\text {ion }} \gtrsim \alpha_{x} n_{e} N_{x \text {,tot }}$, where $\alpha_{x}$ is the recombination coefficient and $n_{e}$ is the electron density in the shell. We find

$$
\begin{aligned}
J_{\text {ion }} \gtrsim & 5 \times 10^{-31}\left(\frac{n_{e}}{\bar{n}_{b}}\right)\left(\frac{f_{*}}{0.1} \frac{126 \mathrm{M}_{\odot}}{\omega_{\mathrm{SN}}} \frac{\Omega_{b} / \Omega_{m}}{0.05 / 0.3} \frac{M_{h}}{10^{8} \mathrm{M}_{\odot}}\right) \\
& \times\left(\frac{Y_{x}}{0.1 \mathrm{M}_{\odot}} \frac{12}{A_{x}}\right)\left(\frac{10 \mathrm{kpc}}{R}\right)^{2}\left(\frac{10}{1+z}\right)^{3}\left(\frac{0.0245}{\Omega_{b} h^{2}}\right)(1,6)
\end{aligned}
$$

in units of $\operatorname{erg~s}^{-1} \mathrm{~cm}^{-2} \mathrm{~Hz}^{-1} \mathrm{sr}^{-1}$ and where $\bar{n}_{b}$ is the mean baryon density of the IGM. The electron density is difficult to constrain. The standard jump conditions for an isothermal shock give a shell baryon density $n_{\mathrm{sh}} \sim \mathcal{M}^{2} \Delta \bar{n}_{b}$, where $\Delta$ is the local overdensity through which the shell travels. However, the shell initially plows through highly overdense material within the halo $(\Delta \gtrsim 200)$ at a very high velocity. Later, the shell thickness can only increase at the sound speed and it may not have had sufficient time to expand to its equilibrium structure.

We now estimate the extragalactic background over the range $\sim 7-13 \mathrm{eV}$, which includes photons able to ionize $\mathrm{C}, \mathrm{Si}$, and $\mathrm{Fe}$. We assume that the emissivity of a typical galaxy obeys $\varepsilon \propto \nu^{-\alpha}$ and is proportional to the mass of the galaxy. The volume emissivity of photons is then

$$
j(\nu, z)=2 \pi \alpha \hbar N_{\text {ion }} f_{*} n_{H}^{0}\left(\frac{\nu}{\nu_{H}}\right)\left|\frac{d z}{d t}\right|\left|\frac{d F_{\text {coll }}}{d z}\right|,
$$

where $F_{\text {coll }}$ is the fraction of collapsed baryons in galaxies and where we have normalized the emission spectrum to the number of photons per baryon in stars emitted above the ionization threshold of hydrogen $\nu_{H}, N_{\text {ion }} \sim 4000$ (Barkana \& Loeb 2001). The extragalactic background is (Haiman, Rees, \& Loeb 1997)

$$
J_{\nu}(z)=\frac{1}{4 \pi} \int j\left(\nu_{z}, z^{\prime}\right) e^{-\tau\left(z^{\prime}\right)} d \ell\left(z^{\prime}\right),
$$

where $\nu_{z}=\nu\left[\left(1+z^{\prime}\right) /(1+z)\right], \tau\left(z^{\prime}\right)$ is the IGM optical depth at $\nu_{z}$, and $\ell$ is the proper length. Below $\nu_{H}$, the only sources of significant opacity are the Lyman lines of hydrogen. For a given observed frequency $\nu$ at $z$, the universe is thus optically thin back to the point $z_{\text {on }}$ at which this radiation would have blueshifted into the nearest Lyman line (denoted here as Lyx),

$$
1+z_{\mathrm{on}}=\frac{\nu_{\mathrm{Ly} x}}{\nu}(1+z) \text {. }
$$

Thus (in units of erg s $\mathrm{cm}^{-2} \mathrm{~Hz}^{-1} \mathrm{sr}^{-1}$ )

$$
\begin{aligned}
J_{\text {ion }}(z)= & 1.3 \times 10^{-21} \alpha\left(\frac{f_{*}}{0.1} \frac{N_{\text {ion }}}{4000} \frac{\Omega_{b} h^{2}}{0.0245}\right)\left(\frac{\nu_{\text {ion }}}{\nu_{H}}\right)^{-\alpha} \\
& \times \int_{z}^{z_{\text {on }}} d z^{\prime}\left(\frac{1+z}{1+z^{\prime}}\right)^{\alpha}\left|\frac{d F_{\text {coll }}}{d z}\right| .
\end{aligned}
$$

For carbon, the integral is typically $\left(\sim 10^{-5}, 3 \times 10^{-4}, 2 \times\right.$ $10^{-3}, 10^{-2}$ ) for $z=20,16,12$, and 8 , with weak dependence on the spectral slope. The background for $\mathrm{Fe}$ and $\mathrm{Si}$ is a few times larger. Comparing equations (16) and (20), we see that so long as the shell is neutral $\left(n_{e} \ll n_{\mathrm{sh}}\right)$ or the shell is not extraordinarily overdense, the extragalactic background will easily ionize these elements to C II, Si II, and Fe II.

By definition, the extragalactic background cannot ionize hydrogen in the shell until after cosmological reionization. We now consider whether the host galaxy can ionize the shell. According to the starburst models of Leitherer et al. (1999), the number of ionizing photons produced per unit time by an instantaneous starburst with a Salpeter IMF and metallicity $Z=0.05 Z_{\odot}$ (the lowest metallicity they calculated) a time $t$ after the burst is

$$
\frac{d N_{\gamma}}{d t} \sim 10^{51.1}\left(\frac{t}{10^{7} \mathrm{yr}}\right)^{-4}\left(\frac{f_{*} M_{g}}{10^{6} \mathrm{M}_{\odot}}\right) \mathrm{s}^{-1}
$$


provided that $t \gtrsim 10^{7}$ yr. Here $f_{*} M_{g}$ is the stellar mass produced in the starburst. Of these, only a fraction $\chi$ can escape the host galaxy. This escape fraction is poorly constrained at present but is likely $\lesssim 10 \%$ (Leitherer et al. 1995; Wood \& Loeb 2000; Dove, Shull, \& Ferrara 2000; Steidel, Pettini, \& Adelberger 2001).

As above, we can calculate the column depth of ionized hydrogen $N_{\mathrm{HII}}$ by balancing the number of ionizations and recombinations per second. The recombination rate again depends on the shell density. $N_{\mathrm{HII}}$ will be largest for the smallest $n_{\mathrm{sh}}$; we therefore assume pessimistically that $n_{\mathrm{sh}} \sim \mathcal{M}^{2} \bar{n}_{b}$. We compare $N_{\mathrm{HII}}$ to the total column in the shell $N_{\mathrm{sh}}$, assuming that the swept up matter dominates the shell mass. We then find, for an instantaneous starburst, that

$$
\begin{aligned}
\frac{N_{\mathrm{HII}}}{N_{\mathrm{sh}}} \sim 10^{-3} & \left(\frac{f_{*}}{0.1} \frac{\chi}{0.1} \frac{M_{h}}{10^{8} \mathrm{M}_{\odot}}\right)\left(\frac{20 \mathrm{kpc}}{R}\right)^{3}\left(\frac{10}{\mathcal{M}^{2}}\right) \\
& \times\left(\frac{10^{8} \mathrm{yr}}{t}\right)^{-4}\left(\frac{10}{1+z}\right)^{6}\left(\frac{0.05}{\Omega_{b}} \frac{0.3}{\Omega_{0}}\right)\left(\frac{0.7}{h}\right)_{22)}^{4}
\end{aligned}
$$

We have confirmed the approximate validity of this simple estimate over the range of densities and spectra of interest with CLOUDY (Ferland 2001). Thus, once an appreciable time has elapsed after the starburst, the shells remain neutral. However, if active star formation persists, $d N_{\gamma} / d t \sim$ $10^{53.2}\left(\dot{M}_{*} / \mathrm{M}_{\odot} \mathrm{yr}\right) \mathrm{s}^{-1}$ (Leitherer et al. 1999). The shell could then in principle remain partially ionized for a longer period. If the shells fragment or if the metals otherwise reside in clouds, the densities will increase beyond $\mathcal{M}^{2} \bar{n}_{H}$, making ionization even more difficult. In this case, the ionization balance would also depend on the size of the clouds, parameterized in our model by $C_{A}$.

Observations of nearby starburst winds and LBG outflows find strong absorption from low ionization states (Heckman et al. 2000; Pettini et al. 2002). In all of these systems, the starburst is still active and hence $d N_{\gamma} / d t$ is large. In the old winds that provide most of the absorption lines, the arguments above show that low ionization states should be even more important. In the following we therefore focus on low ionization states, and we assume that $C_{m}=1$. While this is obviously a naive approximation, our results can easily be rescaled by equation (14). For completeness, we also show some results for ions appropriate to a "warm" medium, C IV and Si IV. These ions may be appropriate if metals are advected to the shell but do not mix efficiently with the swept-up IGM, thus remaining at the interface between the cool shell and the hot cavity. Lines from these ions are prominent in LBG winds (Pettini et al. 2002; Shapley et al. 2002).

If, however, the metals remain locked in the hot bubble cavity, our assumptions will break down. This medium has density $\sim f_{m} \bar{n}_{H}$ and is easily ionized by even trace amounts of star formation in the host. More importantly, the gas in this phase has typical temperatures $T \sim 10^{5.5}-10^{6.5} \mathrm{~K}$ and thus is collisionally ionized to very high ionization states that lack rest-frame optical or ultraviolet absorption lines. Fortunately, any metals carried by the hot wind medium will eventually be advected to the shell, where cooling and mixing will occur (see \$3.2).

\section{RESULTS}

With the wind model described in $\S 2$ and the metal characteristics outlined in $\S 3$, we can now calculate the consequences of metal pollution from galactic winds. We list the parameters of our model, along with their standard choices, in Table 2. Unless otherwise specified, all curves shown assume these values. We also consider two halo cooling channels: atomic hydrogen cooling, which allows halos with $T_{\mathrm{vir}} \gtrsim 10^{4} \mathrm{~K}$ to form stars, and molecular hydrogen cooling, which reduces the threshold to $T_{\mathrm{vir}} \gtrsim 400 \mathrm{~K}$. In the former case, we assume a Scalo (1998) IMF applies, while in the latter we assume a VMS IMF. This choice in turn determines $\omega_{\mathrm{SN}}, E_{51}$, and $Y_{x}$. For $\mathrm{H}_{2}$ cooling, we set $\omega_{\mathrm{SN}}=462 \mathrm{M}_{\odot}$ and $E_{51}=10$.

We present the results of our model in the following sections. First we describe the observable characteristics of individual winds in $\S 4.1$. We then discuss the global evolution of the metal filling factor in $\S 4.2$. Finally, we present our predictions for absorption line statistics in $\S 4.3$.

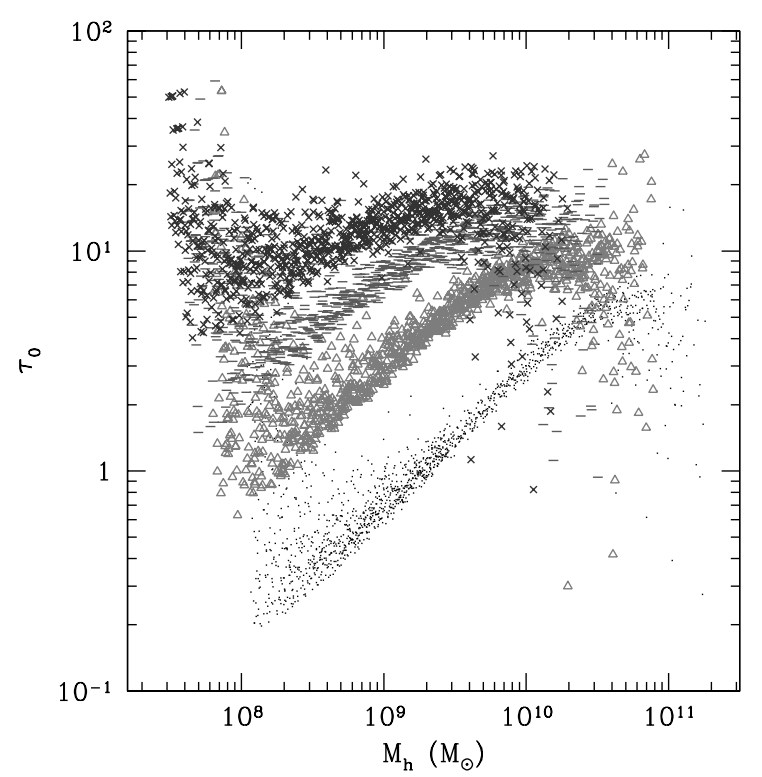

FIG. 3.- Central optical depth $\tau_{0}$ of the O I $\lambda 1302$ feature as a function of halo mass $M_{h}$ for our standard parameter choices. Crosses, horizontal lines, triangles, and points refer to $z=20,16,12$, and 8 , respectively.

\subsection{Wind Characteristics}

Absorption lines are characterized by three basic parameters (of which two are independent): the line width $b$, the central optical depth $\tau_{0}$, and the equivalent width $W$. In our model, we set $b=v_{\text {exp }}$ (see Figure 2). Although we present our results exclusively in terms of $W$, it is also useful to examine the central optical depth $\tau_{0}$ of the absorption systems. In a real observation, $\tau_{0}$ helps to determine the ease with which each line can be identified. It also indicates where saturation becomes important (and hence where equations [13] and [14] break down). The typical column density of an ion $x$ through (each side of) the wind per velocity interval is

$$
N_{x}(u)=\frac{N_{x, \text { tot }}}{\sqrt{\pi} b} e^{-u^{2} / b^{2}},
$$

where $u$ is the velocity relative to $v_{\exp }$. The optical depth at line center is then

$$
\begin{aligned}
\tau_{0}= & \frac{\pi e^{2} f_{\mathrm{osc}} \lambda_{m}}{m_{e} c} \frac{N_{x, \text { tot }}}{\sqrt{\pi} b} \\
= & 0.2\left(\frac{f_{\mathrm{osc}}}{0.05} \frac{Y_{x}}{0.5 \mathrm{M}_{\odot}} \frac{16}{A_{x}} \frac{\lambda_{m}}{1300 \AA}\right)\left(\frac{50 \mathrm{~km} \mathrm{~s}^{-1}}{b}\right) \\
& \times\left(\frac{f_{*}}{0.1} \frac{126 \mathrm{M}_{\odot}}{\omega_{\mathrm{SN}}} \frac{\Omega_{b} / \Omega_{0}}{0.05 / 0.3} \frac{M_{h}}{10^{9} \mathrm{M}_{\odot}}\right)\left(\frac{20 \mathrm{kpc}}{R}\right)^{2} .
\end{aligned}
$$


We show $\tau_{0}$ as a function of halo mass for several redshifts in Figure 3. We have chosen the O I $\lambda 1302$ transition for this plot because it is the strongest low ionization absorber in our model. Because $R \propto M_{h}^{1 / 5}$ while $b$ is roughly constant in our model, $\tau_{0} \propto M_{h}^{3 / 5}$ below $M_{\max } . \tau_{0}$ increases with $z$ because wind bubbles are more compact at higher redshifts and hence the typical column density $N_{x}$ is much larger. We see that line saturation is not important for the low mass-halos that contribute most of the filling factor at $z \lesssim 12$, but it cannot be neglected at higher redshifts. At a given $z$, the minimum $\tau_{0}$ is determined by the smallest halos that can collapse to form stars (fixed in this case by the atomic cooling threshold).

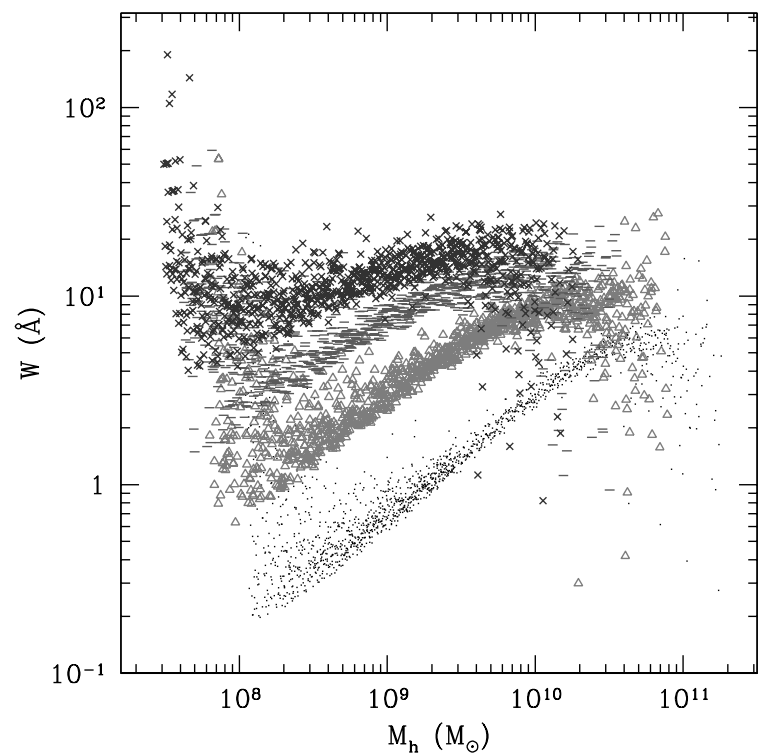

FIG. 4.- Observed equivalent width $W$ of the O I $\lambda 1302$ feature as a function of halo mass $M_{h}$ for our standard parameter choices. Crosses, horizontal lines, triangles, and points refer to $z=20,16,12$, and 8 , respectively.

The observed equivalent width $W$ is

$$
W=(1+z) \int\left[1-e^{-\tau(\lambda)}\right] d \lambda .
$$

If the line is not saturated, this expression may be written

$$
\begin{aligned}
W \approx & 0.8 \AA\left(\frac{f_{\text {osc }}}{0.05} \frac{Y_{x}}{0.5 \mathrm{M}_{\odot}} \frac{16}{A_{x}}\right)\left(\frac{\lambda_{m}}{1300 \AA}\right)^{2}\left(\frac{1+z}{10}\right) \\
& \times\left(\frac{f_{*}}{0.1} \frac{126 \mathrm{M}_{\odot}}{\omega_{\mathrm{SN}}} \frac{\Omega_{b} / \Omega_{0}}{0.05 / 0.3} \frac{M_{h}}{10^{9} \mathrm{M}_{\odot}}\right)\left(\frac{20 \mathrm{kpc}}{R}\right)^{2}
\end{aligned}
$$

We show $W$ as a function of halo mass in Figure 4 (again for O I $\lambda 1302$ ). Typical equivalent widths increase from $\sim 0.5-$ $10 \AA$ between $z=8$ and $z=20$. $W$ increases with redshift both because of stretching by the cosmological redshift and because the wind bubbles are smaller at these times. When line saturation can be neglected, $W \propto b \tau_{0} \propto M_{h}^{3 / 5}$. As the lines become saturated, the relationship flattens. Interestingly, Figure 4 suggests that a line of a given equivalent width can be associated with a halo in a fairly narrow mass range, particularly at lower redshifts. This is essentially a consequence of the relatively small dispersion in wind bubble sizes for a fixed set of parameters and our assumption that $f_{*}$ and $f_{\text {esc }}$ do not vary systematically with halo mass. As a result, $N_{x}$ is well-determined given the halo mass. There are some recent indications that $f_{*} \propto M_{h}^{2 / 3}$
(Kauffmann et al. 2002; Dekel \& Woo 2002). If so, this would steepen the $M_{h}-W$ relation while preserving the one-to-one correspondence.

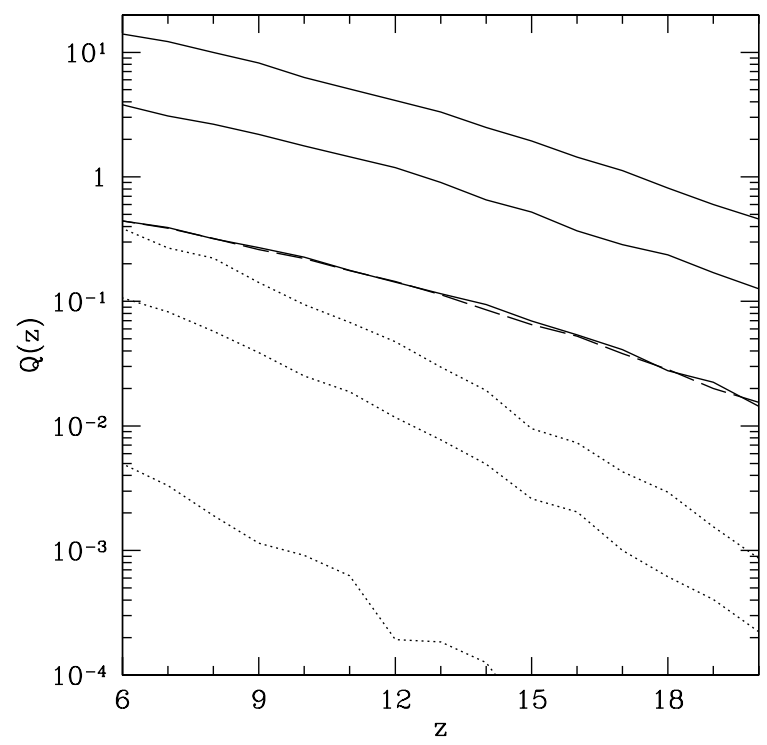

FIG. 5.- Filling factor of polluted regions as a function of redshift. Solid curves assume $\mathrm{H}_{2}$ cooling and a VMS IMF, while dotted curves assume atomic cooling and a Scalo IMF. Each set has $f_{*}=0.5,0.1$, and 0.01 from top to bottom; other parameters take their standard values. The dashed curve assumes $\mathrm{H}_{2}$ cooling with $f_{*}=0.1$ and $E_{51}=1$.

\subsection{Filling Factor}

To calculate the filling factor of the wind bubbles, we compute

$$
Q(z)=\int_{M_{\min }(z)}^{\infty} d M \frac{d n}{d M} V(M, z),
$$

where $V(M, z)$ is the comoving volume of the wind produced by a halo of mass $M$ at a redshift $z$ and $d n / d M$ is the halo mass function (Press \& Schechter 1974; Sheth \& Tormen 1999). Note that we do not include photoheating due to reionization in determining $M_{\min }$. We therefore likely overestimate $Q(z)$ for $z<6$, but we include these results for comparison with previous work. Because the star formation history determines the wind size, we perform the integral using a Monte Carlo algorithm. We chose the mass sampling by requiring that our results converge to $\lesssim 10 \%$ between trials. ${ }^{6}$ Because winds escape small halos most easily, $Q(z)$ is dominated by winds from low-mass halos (in most of our calculations, winds with $M_{h} \lesssim 10 M_{\min }(z)$ account for $\sim 75 \%$ of the total polluted volume); in practice, the integral need only extend to some maximum mass $M_{\max }$ (see $\S 2.2$ ). The principal shortcoming of this model is that it neglects the overlap of wind bubbles; thus, we can have $Q(z)>1$. If sources are randomly distributed, the true filling factor will be $\Phi=1-\exp [-Q(z)]$. However, the relationship between $Q(z)$ and the true filling factor cannot be so easily quantified if sources are clustered (see $\$ 5)$.

Figures 5 and 6 show $Q(z)$ in our model. In Figure 5, we compare $Q(z)$ for $\mathrm{H}_{2}$ cooling (solid curves) and atomic cooling (dotted curves). Within each set, the three curves correspond to $f_{*}=0.01,0.1$, and 0.5 , from bottom to top. All other parameters have their standard values. We see that, in the atomic cooling

${ }^{6}$ For $f_{*} f_{\text {esc }} \lesssim 5 \times 10^{-3}$, stochastic fluctuations are large and the convergence is somewhat worse; see Figure 5 for an example. 
scenario, $Q(z=12) \approx 1 \%$ and $Q(z=6) \approx 10 \%$ (for $f_{*}=0.1$ ). The filling factor increases rapidly with cosmic time because of the increasing fraction of baryons in collapsed objects and because the winds have considerably more time to expand at low redshifts, a point emphasized by Aguirre et al. (2001c). By allowing less massive halos to form stars and by injecting an order of magnitude more energy per supernova, the $\mathrm{H}_{2}$ cooling scenario leads to a much larger filling factor: with $f_{*}=0.1$, this model has $Q(z=12) \approx 1$. The dashed line (nearly obscured by the lowest solid line) shows the $\mathrm{H}_{2}$ cooling scenario with $f_{*}=0.1$ and $E_{51}=1$; thus, even if $\mathrm{H}_{2}$ cooling creates "normal" massive stars the enrichment process is still much more efficient than with pure atomic cooling. At very high redshifts, the minimum halo mass in the atomic cooling scenario is above the nonlinear mass scale in the Sheth \& Tormen (1999) mass function. In this regime, the halo abundance is exponentially suppressed; therefore, $Q(z)$ for $\mathrm{H}_{2}$ cooling greatly exceeds that for atomic cooling. The gap closes (though it is still substantial) as $M_{\min }$ falls below the nonlinear mass scale at lower redshifts. Our results for $Q(z)$ in the atomic cooling scenario are very similar to those of Scannapieco, Ferrara, \& Madau (2002), even though they use a more sophisticated algorithm to determine star formation efficiencies and halo clustering. We find significant differences, however, between their "Population III" scenario and our $\mathrm{H}_{2}$ cooling scenario. The disparity is likely caused by their requirement that $\mathrm{H}_{2}$ cooling occurs before disk formation which imposes a much more stringent mass threshold than ours, and because we assume that VMS supernovae are more energetic.

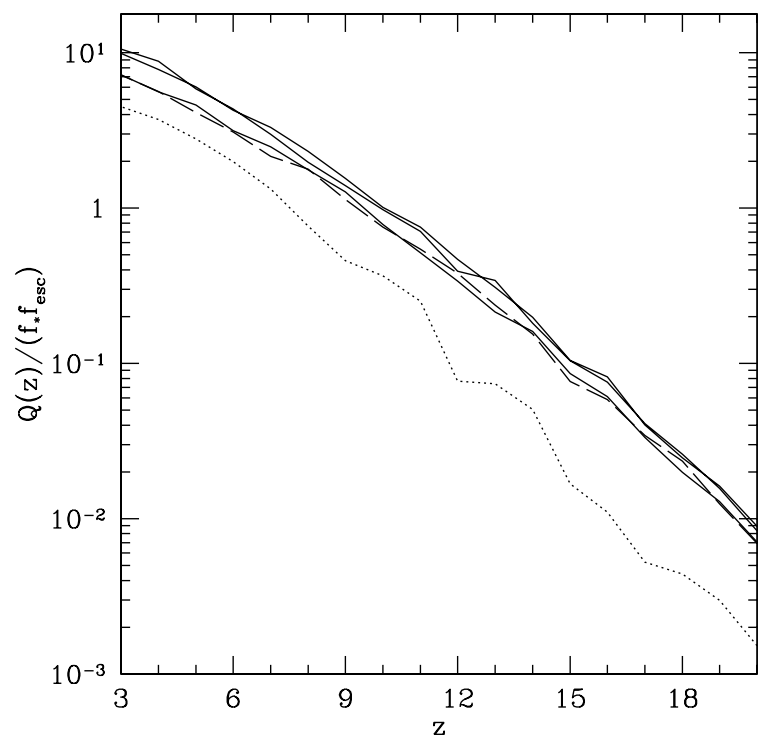

FIG. 6.- Filling factor of enriched regions as a function of redshift normalized to $f_{*} f_{\mathrm{esc}}, Q(z) /\left(f_{*} f_{\mathrm{esc}}\right)$. The three solid curves show $f_{\mathrm{esc}}=1,0.25$, and 0.1 with $f_{*}=0.1$. The dashed and dotted curves assume $f_{*}=0.5$ and $f_{*}=0.01$, respectively, with $f_{\mathrm{esc}}=0.25$. All other parameters take their standard values.

Figure 6 plots $Q(z) /\left(f_{*} f_{\text {esc }}\right)$ for the atomic cooling scenario. We have thus normalized $Q(z)$ by the factor controlling the energy output in the outflows. This normalized filling factor is constant (to within a factor $\sim 2$ ) at a fixed redshift as long as $f_{*} f_{\text {esc }} \gtrsim 5 \times 10^{-3}$ (c.f., Scannapieco, Ferrara, \& Madau 2002). As discussed in $\$ 2.2$, our model yields $R \propto E^{0.35}$ at a fixed mass $M_{h} \ll M_{\max }$. Thus, $Q(z) /\left(f_{*} f_{\text {esc }}\right)$ should be nearly constant so long as the halos that contribute most to the filling factor (i.e., the first decade in mass above $M_{\min }$ ) are well below this mass threshold for efficient escape. The proportionality is broken for $f_{*} f_{\text {esc }} \lesssim 5 \times 10^{-3}$ because the number of halos hosting substantial winds begins to depend on $f_{*} f_{\text {esc }}$.

\subsection{Line Statistics}

Unfortunately, the volume filling factor $Q(z)$ is difficult to observe, requiring sophisticated pixel-by-pixel analyses (e.g., Aguirre, Schaye, \& Theuns 2002). It is therefore more useful to consider the statistics of absorption lines. The number of systems intersected per redshift interval with an observed equivalent width $W$ greater than some threshold $W_{0}$ along a random line of sight is

$$
\frac{d N\left(>W_{0}\right)}{d z}=\frac{d r}{d z} \int_{M_{\min }(z)}^{\infty} d M \frac{d n}{d M} A(M, z) \Theta\left(W-W_{0}\right),
$$

where $A(M, z)$ is the comoving cross-sectional area of the wind, $d r / d z$ is the comoving distance per unit redshift, and $\Theta\left(W-W_{0}\right)$ is unity if the equivalent width of a typical line of sight through the wind is greater than $W_{0}$ and zero otherwise. Again, our model neglects halo clustering (see $\$ 5$ for a discussion of its effects). We could in principle choose random impact parameters for each bubble; however, given the approximations we have already made, we consider this extra layer of complexity to be unnecessary. Note that equation (28) assumes a single absorption line per halo. In the ideal case, each intersected wind bubble would cause 2 lines separated by $\sim 2 v_{\exp }$. However, if the velocity resolution of the observation is lower than this value, the two lines would appear as a single feature, albeit with an equivalent width up to twice that which we assume. The two lines will also merge if $b>v_{\text {exp }}$. For clarity, we therefore assume that only one line is produced per halo.

Another important quantity is a measure of the total flux per redshift interval absorbed by a single transition (neglecting overlap of the lines),

$$
W_{\mathrm{tot}}=\int W \frac{d}{d W}\left[\frac{d N(>W)}{d z}\right] d W .
$$

If $W_{\text {tot }} \ll \lambda_{m}$, lines will be isolated and easily separated. If $W_{\text {tot }} \sim \lambda_{m}$, lines blend together and absorb a large fraction of the flux from the background source.

Figure 7 shows the evolution of $d N(>W) / d z$ with redshift. Here we show results for O I $\lambda 1302$, the strongest line in the atomic cooling scenario. The curves show line statistics at $z=20$ (dotted line), $z=16$ (short-dashed line), $z=12$ (longdashed line), and $z=8$ (solid line). Each assumes our standard parameter set. Clearly, the number of lines intersected depends strongly on redshift, particularly for weak lines (increasing from $\sim 0.25$ to $\sim 40$ with $W>0.5 \AA$ per redshift interval from $z=20$ to $z=8$ ). Two factors contribute to this increase: the winds are more compact at higher redshifts (see Figure 1) and the fraction of baryons in star-forming galaxies is much smaller at higher redshifts. The shape of the curves are easy to understand. As shown in Figure 4, a given $W$ roughly corresponds to halos of a particular mass at fixed $z$. Thus, at each redshift, $d N(>W) / d z$ is a measure of the (cumulative) halo mass function weighted by $R^{2}$. The "saturation" at small $W$ is simply a result of the minimum halo mass for star formation $M_{\min }$. Figure 7 also shows how $W_{\text {tot }}$ evolves with redshift. Recalling that $\mathrm{O} \mathrm{I}$ is the strongest line we consider, line blanketing should not be significant for $f_{*}=0.1$ in the atomic cooling scenario. 


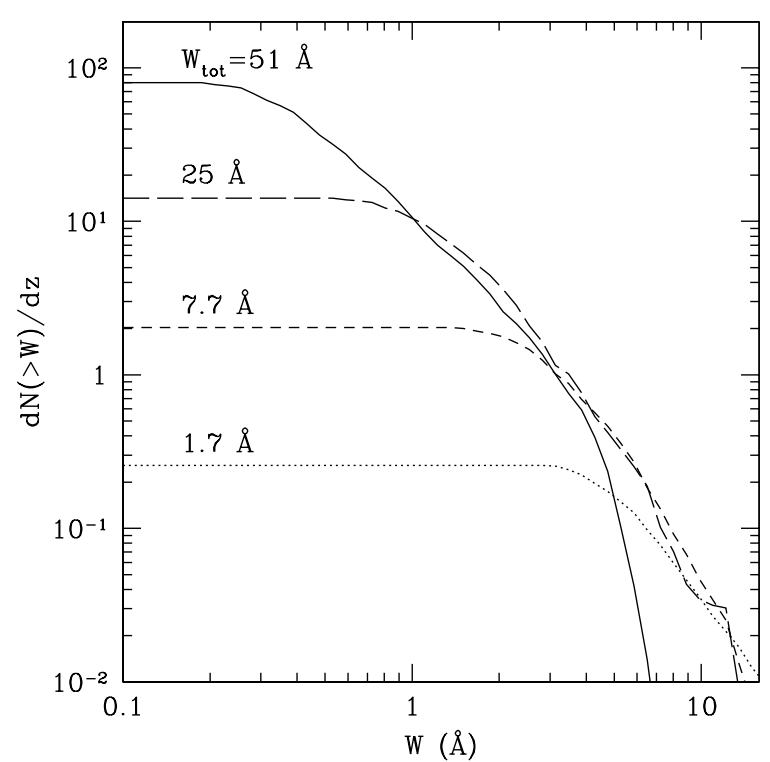

FIG. 7.- Number of intersections per redshift interval above a given equivalent width threshold for O I $\lambda 1302$. From top to bottom, curves refer to $z=8,12,16$, and 20. All parameters take their standard values. We also list $W_{\text {tot }}$ (see equation [29]) for each line.

Figure 8 shows the $\mathrm{O}$ I statistics for the atomic cooling scenario at $z=8$. From top to bottom, the three solid curves assume $f_{*}=0.5,0.1$, and 0.01 , with $f_{\text {esc }}=0.25$. The two dashed curves assume $f_{\text {esc }}=1$ and 0.1 (top and bottom at the left, respectively), with $f_{*}=0.1$. All other parameters are standard. Recall that $Q(z) \propto f_{*} f_{\text {esc }}$; clearly, the dependence of the line statistics on these parameters is not degenerate. Essentially, absorption lines give information about both the extent of the enriched IGM and the level of enrichment. Recall that $f_{\text {esc }}$ affects only the amount of energy powering the wind, while $f_{*}$ affects both the energy input into the wind and the total mass of metals produced. Thus, an increase in $f_{\text {esc }}$ increases the number of weak lines but decreases the number of strong lines, because the metals become more dilute. An enhanced star formation efficiency increases the number of lines of all strengths. The total absorption $W_{\text {tot }}$ strikingly reveals the difference. As shown in Figure $8, W_{\text {tot }}$ increases rapidly with $f_{*}$. However, it varies only slowly with $f_{\text {esc }}$, increasing from $41 \AA$ to $57 \AA$ between $f_{\text {esc }}=0.1$ and $f_{\text {esc }}=1$. Note that if $f_{*}$ is very high at this redshift, line blanketing from the many possible transitions becomes important.

The greatly increased filling factor of polluted regions for $\mathrm{H}_{2}$ cooling compared to atomic cooling (see Figure 5) suggests that these two scenarios may be distinguished through observations of line statistics. Figure 9 shows that this is indeed the case. We show the statistics for four different transitions (O I $\lambda 1302$; long-dashed lines; C II $\lambda 1334$, solid lines; Fe II $\lambda 2344,{ }^{7}$ dotted lines; Si II $\lambda 1304$, short-dashed lines) at $z=20$ and $z=12$. The upper set of curves in each panel assumes $\mathrm{H}_{2}$ cooling and a VMS IMF, while the lower set assumes atomic cooling and a Scalo IMF. All other parameters are standard. We also list $W_{\text {tot }}$ for the O I $\lambda 1302$ transition in each case. The difference between $d N / d z$ for the two models (a factor $\sim 1100$ at $z=20)$ is even more dramatic than the difference in $Q(z)$ (a factor $\sim 500$ at $z=20$ ). This occurs because of the rapidly increasing mass function at small masses, which allows many more individual halos to produce winds if $\mathrm{H}_{2}$ cooling is permitted; the line statistics are more sensitive to the number of starforming halos than is $Q(z)$ because they depend most strongly on the total number of individual winds that can in principle be intersected. However, because the additional halos are small, they generally produce weak lines. Therefore the enhancement of weak lines is much larger than the enhancement of strong lines (this being particularly evident at $z=12$ ). Interestingly, $W_{\text {tot }}$ is very large for all redshifts in the $\mathrm{H}_{2}$ cooling scenario. Thus a large fraction of the background source flux could be absorbed by overlapping metal lines in this scenario, extending the apparent Gunn-Peterson absorption to much higher wavelengths than otherwise expected.

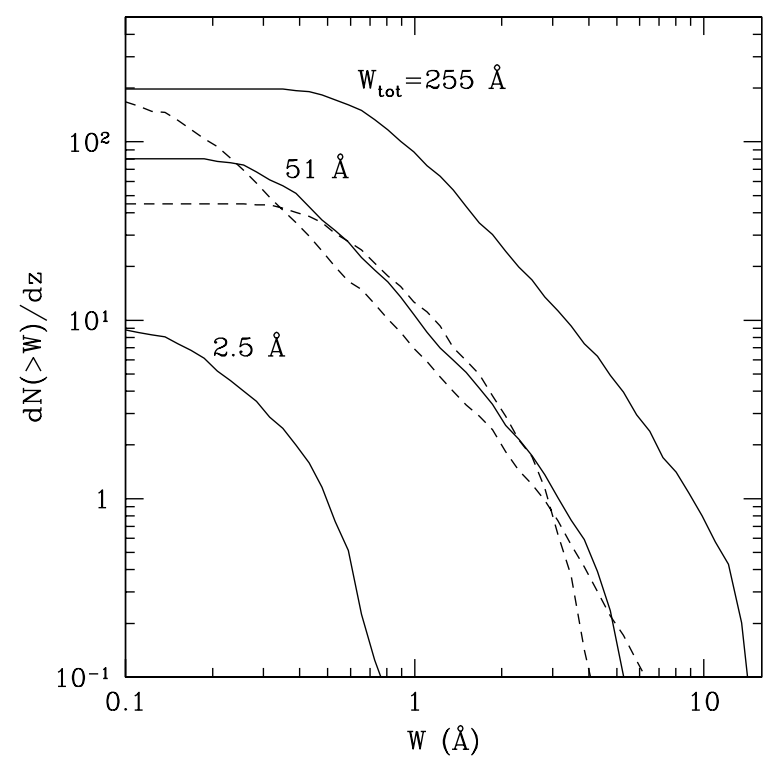

FIG. 8.- Number of intersections per redshift interval above a given equivalent width threshold at $z=8$ for the O I $\lambda 1302$ transition. The solid lines assume $f_{*}=0.5,0.1,0.01$, from top to bottom, and $f_{\text {esc }}=0.25$. The dashed lines assume $f_{\mathrm{esc}}=1$ and 0.1 , from top to bottom at left, and $f_{*}=0.1$. All other parameters are standard. We also list $W_{\text {tot }}$ (see equation [29]) for the solid curves. $W_{\text {tot }}$ varies only slowly with $f_{\text {esc }}$ (see text).

We also see the effects of a VMS IMF in Figure 9. Although $\omega_{\mathrm{SN}}$ varies by less than a factor $\sim 4$ between the two models (and is actually larger for a VMS IMF), the explosion energy for the VMS IMF is an order of magnitude larger than for a Scalo IMF. (Note, however, that even if $E_{51}=1$ for VMS stars, the differences between the two scenarios are large; see Figure 5). The different yields of the two IMFs also cause differences in the relative line strengths. For a Scalo IMF, the strongest features are $\mathrm{O}$ I and $\mathrm{C}$ II, simply because these are the most abundant elements produced in Type II supernovae. Pair instability supernovae, on the other hand, completely disrupt the star and therefore expel heavy elements much more efficiently (Heger \& Woosley 2002; see Table 1). As a result, the strongest features in VMS winds are $\mathrm{O}$ I and Fe II, with $\mathrm{C}$ II being the weakest feature.

We argued in $\S 3.3$ that the low ionization states of metals are likely to be most important, but it is also possible that many metals will be in relatively high ionization states. Figure 10 shows $d N(>W) / d z$ at $z=8$. The thick curves show low ion-

\footnotetext{
${ }^{7}$ We have selected this line from the several available Fe II transitions because it has an "average" oscillator strength. The strongest line, Fe II $\lambda 2382$, will have an equivalent width $\sim 3$ times greater than that shown.
} 
ization states (as in Figure 9) while the thin curves show high ionization states (C IV $\lambda 1548$, solid line; Si IV $\lambda 1394$, shortdashed line). In each case we assume $C_{m}=1$. We see that high ionization states actually provide a slightly stronger signal than the low ionization states; our previous predictions are therefore conservative in this sense. However, if the metals are even more highly ionized, no observable transitions will remain with $\lambda_{m}>\lambda_{\text {Ly } \alpha}$.

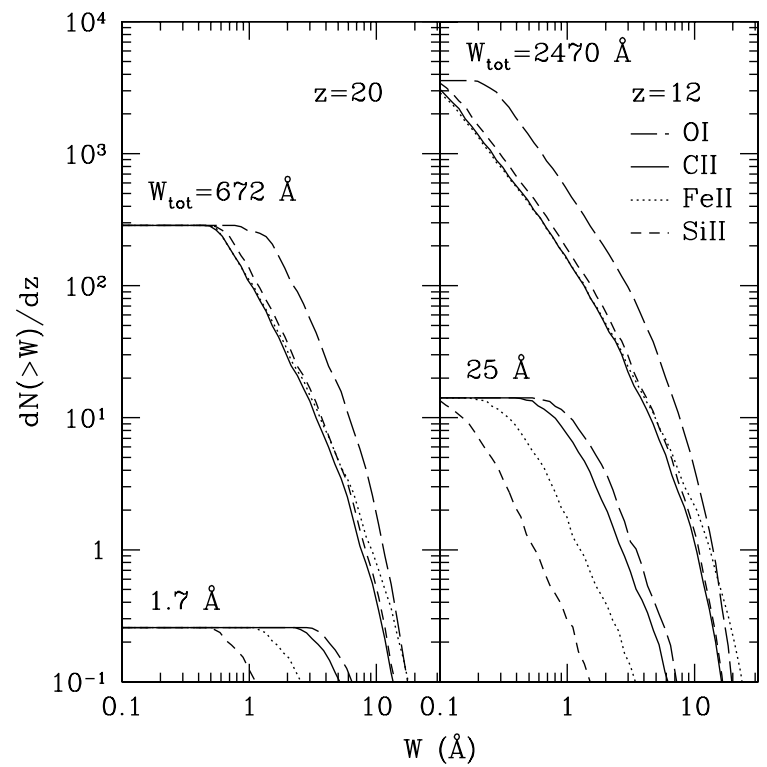

FIG. 9.- Number of intersections per redshift interval above a given equivalent width threshold. In each panel, the bottom set of curves assumes atomic cooling while the top set allows $\mathrm{H}_{2}$ cooling. All other parameters take their standard values. We also list $W_{\text {tot }}$ (see equation [29]) for the O I $\lambda 1302$ transition in each scenario.

Finally, Figure 11 shows the effect of varying other parameters of the outflow model. All the curves assume our standard parameter choices with the following exceptions: $f_{d}=1$ (dotted line), $f_{\mathrm{sw}}=1$ (dot-dashed line), and no disk formation (dashed line). We see that the uncertainty due to model details at $z=8$ is $\lesssim 30 \%$. The uncertainties in $Q(z)$ are somewhat larger $(\sim 50 \%)$, because the integrand of equation (27) is proportional to $R^{3}$, while $d N / d z$ is proportional to the cross-sectional area $R^{2}$. Thus our predictions for line statistics are fairly insensitive to the detailed wind parameters. Note, however, that the uncertainties increase with redshift, reaching a factor $\sim 2$ by $z=20$ for atomic cooling. At high redshifts, the winds have spent a larger fraction of their expansion within the halo and are hence more sensitive to its structure. In contrast, at low redshifts most of the expansion takes place in the IGM and is independent of assumptions about the halo structure. Model assumptions are much less important in the $\mathrm{H}_{2}$ cooling scenario because the characteristic halo mass (and hence physical scale over which the halo structure matters) is much smaller.

We have examined the absorption by metals in high- $z$ starburst winds seen against a background light source. We allow each star-forming halo to host a wind, with a time-dependent luminosity determined by its star formation history. We use the thin-shell approximation to trace the size of each wind bubble. Our model predicts that LBG winds can reach a proper distance $\sim 100 \mathrm{kpc}$ from the host. It is encouraging that this re- sult roughly matches the observationally inferred distance from LBGs within which $\mathrm{H} \mathrm{I}$ is suppressed (Adelberger et al. 2002). ${ }^{8}$ Our results for the filling factor $Q(z)$ are consistent with previous work (e.g., Scannapieco, Ferrara, \& Madau 2002), but we have emphasized the large uncertainties in the wind modeling and the correspondingly large uncertainty in $Q(z)$. We argue that the statistics of metal absorption lines are a useful way to constrain these parameters. Not only are these statistics directly observable, but they are also less dependent on the wind modeling than $Q(z)$ is. Our predictions are therefore relatively robust, although detailed modeling of the wind structure will be essential for a complete interpretation of the line statistics.

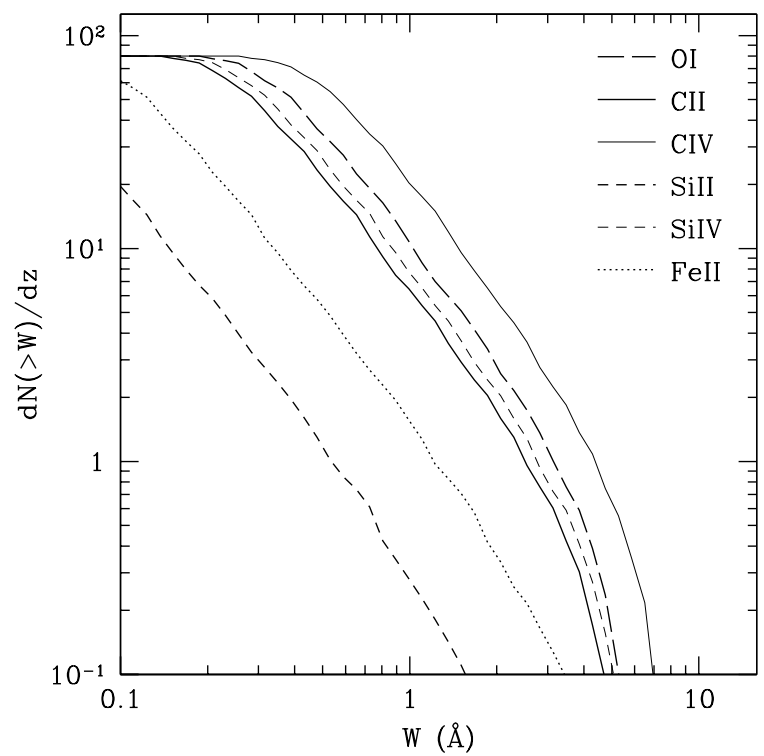

FIG. 10. - Number of intersections per redshift interval above a given equivalent width threshold at $z=8$, assuming our standard parameter choices. Thick solid, long-dashed, short-dashed, and dotted curves are for C II, O I, $\mathrm{Si}$ II, and Fe II, respectively. Thin solid and short-dashed curves are for C IV and Si IV, respectively. In each case we fix $C_{m}=1$.

\section{DISCUSSION}

We estimate that $\sim 40$ O I $\lambda 1302$ lines with equivalent width $W>0.5 \AA$ should be visible per unit redshift at $z=8$, assuming a star formation efficiency $f_{*}=0.1$ and neglecting fragmentation. This decreases to $\sim 0.25$ lines per unit redshift by $z=20$, both because of the smaller fraction of baryons in collapsed objects and because of the brief time that each wind has had to expand by that time. The normalization and shape of the line statistics will primarily constrain $f_{*}$ and $f_{\text {esc }}$, the fraction of supernova energy that powers the wind. Because the total amount of absorption is proportional to the amount of metals ejected, it depends strongly on $f_{*}$ but is nearly independent of $f_{\text {esc }}$. The ratio of strong to weak lines, on the other hand, measures $f_{\text {esc }}$ because that parameter controls the volume over which a fixed metal mass can expand (see Figure 8).

If we allow $\mathrm{H}_{2}$ cooling to occur, then we expect $\sim 250 \mathrm{O}$ I $\lambda 1302$ lines per unit redshift at $z=20$, because this cooling mechanism lowers the threshold halo mass for star formation and increases the explosion energy per supernova. In fact, the combined absorption from the several allowed transitions will eliminate a substantial fraction of the flux from the background

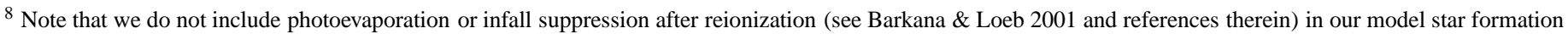
histories. However, LBGs are the most massive objects at $z \sim 3$, for which these processes can be ignored. 


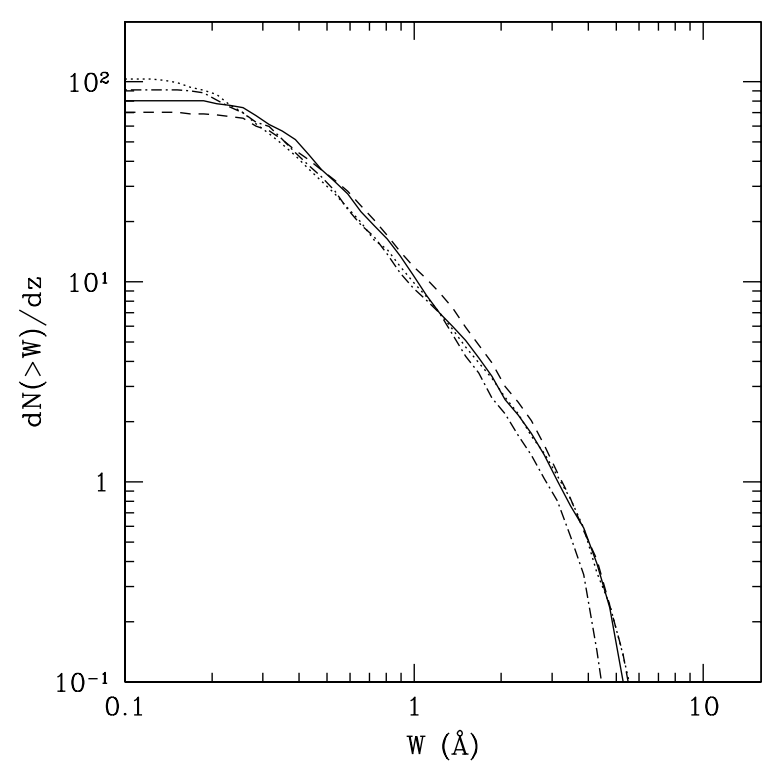

FIG. 11. - Number of intersections per redshift interval above a given equivalent width threshold at $z=8$ for the O I $\lambda 1302$ transition. All curves assume our standard parameter set with the following exceptions: $f_{d}=1$ (dotted curve), no disk formation (dashed curve), and $f_{\mathrm{sw}}=1$ (dot-dashed curve).

source at wavelengths longer than the onset of the GunnPeterson trough. This would be straightforward to detect, but would also make isolating individual lines difficult. The dramatic differences between this scenario and the atomic cooling scenario are large enough that the two should be easily separable even given our uncertainties in $f_{*}$. Metal absorption lines therefore offer a possible route to determine when and if $\mathrm{H}_{2}$ was destroyed throughout the IGM. This transition has important implications for the early growth of cosmic structure (e.g., Wyithe \& Loeb 2002d; Mackey, Bromm, \& Hernquist 2002). The relative strengths of the observed lines contain information about the IMF. In particular, Fe lines will be much more prominent if pair instability supernovae dominate (i.e., if the IMF is biased toward very massive stars).

Metal absorption line studies can shed light on the chemical evolution of the universe. Qian \& Wasserburg $(2001,2002)$ have proposed an important model for the enrichment history of the universe based on abundance patterns in Galactic halo stars. They argue for a "prompt" enrichment phase to a threshold iron abundance $[\mathrm{Fe} / \mathrm{H}] \approx-3$ after which the abundance patterns change markedly; below this threshold, VMS supernovae dominate the enrichment, whereas above it "normal" supernovae dominate. The authors implicitly assume, based on ionic abundances in the Ly $\alpha$ forest (Qian et al. 2002), that the prompt phase uniformly enriches all regions of the IGM to this threshold. Qian et al. (2002) find little evidence for evolution in the elemental abundances in the Ly $\alpha$ forest over the range $z \sim 4.6-0.09$, indicating that the prompt enrichment occurred at $z \gtrsim 5$. Qualitatively, we have shown that an early phase of halo formation through $\mathrm{H}_{2}$ cooling forming VMS stars can pollute large regions of the IGM, lending credence to this assumption. Figure 5 shows that complete enrichment of the entire IGM by this epoch requires fairly efficient star formation $\left(f_{*} \gtrsim 10 \%\right)$ in small halos. In fact, observations of the Ly $\alpha$ forest metallicity do not require complete mixing of metals in the IGM, because these studies are currently only sensitive to absorbers with $N_{H I} \gtrsim 10^{14.5} \mathrm{~cm}^{-2}$, corresponding to moderately overdense regions. Metal enrichment is likely to be confined in islands that correlate with overdense regions where the $\operatorname{Ly} \alpha$ absorption is enhanced. If VMS regions are preferentially clustered near cosmic overdensities, it is plausible that those regions observable as Ly $\alpha$ absorbers with HI column densities $\gtrsim 10^{14.5} \mathrm{~cm}^{-2}$ are enriched while other regions remain pristine. In this case, lower star formation efficiencies would be allowed. We would also expect regions polluted by VMS supernovae and those subsequently polluted by normal supernovae to differ in their abundance patterns. An observational probe of the abundance patterns in the low-density IGM could therefore reveal the era over which the pollution occurred, although any such measurement would be extremely difficult. Note that the Qian $\&$ Wasserburg (2001) model assumes a one-to-one, monotonically increasing relation between metallicity and cosmic time. If mixing is not complete at high redshifts, the transition from "very massive" to "normal" star formation may occur at different times in different regions of the universe. Thus, the nonuniformity of the enrichment process, which metal absorption line studies probe directly, determines the non-uniformity of the transition between different modes of star formation.

We argued that a substantial fraction of the metals are likely to exist in low-ionization states (typically C II, O I, Si II, and Fe II). If, however, the metals exist primarily in higher ionization states, such as C IV and Si IV, then the absorption signal will actually be somewhat easier to observe because the relevant transitions are stronger (see Table 1). Moreover, each of these transitions is a doublet and hence relatively easy to identify unambiguously. If the metals remain in the hot bubble gas with $T \sim 10^{6} \mathrm{~K}$, they will reside in extremely high ionization states (such as $\mathrm{O}$ VI) whose resonant transitions are all blueward of Ly $\alpha$ and therefore unobservable in the high- $z$ IGM.

The largest uncertainty in constraining $f_{*}, f_{\mathrm{esc}}, \mathrm{H}_{2}$ cooling, and the IMF will probably be the covering factor of the enriched material in the wind, $C_{A}$. Meaningful constraints on this factor must await more detailed models of high- $z$ winds, because inferences from observations of local starburst winds may not apply to sources in the early universe. For example, there are indications that outflows from LBGs are approximately spherical (Shapley et al. 2002), unlike the bipolar winds observed in local starbursts. Furthermore, existing observations of absorption by starburst winds and LBG outflows all use the host galaxy as the background light source and hence probe only the early phases of the starburst. Observations using a background source unrelated to the host, such as a quasar or GRB, probe winds of all ages, which may have much different structure. The analysis of Theuns, Mo, \& Schaye (2001) implies that winds at $z \sim 3$ must fragment with $C_{A} \lesssim 0.01$ in order not to overproduce Ly $\alpha$ forest lines. It is, however, unclear whether fragmentation of this degree will occur at the high redshifts we study given the smaller cosmic times available to the winds.

Unfortunately, uncovering the properties of old winds will be difficult. When the host galaxy is used as the background source, the absorption lines themselves can constrain the structure of the wind because saturated lines fix the fraction of the galaxy hidden by the absorbing medium (e.g., Heckman et al. 2000). However, our background sources are pointlike and cannot be used in this way. One strategy to study the outflow structure is to compare the strengths of the low and high ionization components of individual winds. A better strategy is to compare the absorption along neighboring lines of sight to close sources on the sky, such as quasar pairs (e.g., Bechtold et al. 
1994; Crotts et al. 1994) or multiple images of a single gravitationally lensed source (e.g., Rauch et al. 2002). Lensed quasar images are particularly interesting because the lensing probability increases rapidly with quasar redshift (Wyithe \& Loeb 2002a,b). Such studies can determine the projected extent of individual wind systems and the distribution of metal clouds within them.

Our model has two important shortcomings. First, we ignore all effects arising from large-scale structure. If star-forming galaxies are highly clustered (as appears likely at very high redshifts), then their winds will overlap and decrease the filling fraction of metal-enriched material. Clustered sources will also reside in overdense regions of the IGM, further reducing the wind bubble sizes. Qualitatively, both these effects will reduce the number of intersected lines but increase their average strength. Clustering will also increase the variance in the number of absorption lines per unit redshift, because those lines of sight that pass through overdense regions will have greatly increased numbers of intersections. Finally, the filamentary nature of large-scale structure will affect the geometry of the winds. Those winds expanding into filaments will stall earlier, while those expanding into voids will grow more quickly, resulting in anisotropic growth (Theuns et al. 2002). Thus most of the enriched volume will be inside voids, which would tend to increase the filling fraction. For example, with the extreme assumption that all winds expand into a medium with $\rho_{g}$ equal to one-tenth the mean density of the universe, the filling factor of enriched material increases by a factor $\sim 3-5$. On the other hand, if wind sources are embedded in overdense regions, the filling factor decreases even more dramatically because $M_{\max }$ decreases rapidly. Unfortunately, a detailed treatment of clustering and the anisotropic expansion around filaments requires considerably more machinery than is developed here and is best addressed through numerical simulations. We have chosen a similar wind model to Springel \& Hernquist (2002), so our results should help to interpret the effects of the wind model parameterization in such simulations.

The second shortcoming of our model is the long-term fate of the winds. They are likely to fragment due to cooling instabilities or inhomogeneities in the ambient medium. If turbulence is present, the fragments will then mix with the surrounding IGM. Provided that this mixing occurs at approximately the sound speed, our estimates of the total filled volume will not be grossly changed; however, fragmentation could strongly affect the covering fraction of the metals (see above) and their velocity widths.

There are at least two types of astronomical objects that can function as background sources. The most obvious are quasars. Bright quasars have been observed to $z=6.3$ (Fan et al. 2002) and likely extend to $z \sim 10$ (Haiman \& Loeb 2001). However, the characteristic quasar luminosity is expected to decline sharply with increasing redshift (Wyithe \& Loeb 2002c) and it would be difficult to find luminous quasars at much higher redshifts. Quasars also suffer from complicated intrinsic absorption, and isolating weak IGM metal lines may be challenging. Another possibility is to use gamma-ray bursts (GRBs) as the background sources. If GRBs are associated with massive star formation, some likely occur at extremely high redshifts (Lamb

\footnotetext{
${ }^{9}$ See http://www2.keck.hawaii.edu:3636/realpublic/inst/nirspec/nirspec.html.

${ }^{10} \mathrm{See}$ http://www.eso.org/instruments/isaac/.

${ }^{11}$ See http://www.stsci.edu/ngst/.

${ }^{12}$ See http://www.ngst.stsci.edu/nms/index.html.
}

\& Reichart 2000; Bromm \& Loeb 2002). Cosmic time dilation and a favorable $K$-correction imply that, at a given time after the burst in the observer frame, the decrease in brightness with $z$ is mild. Ciardi \& Loeb (2000) show that the $2 \mu \mathrm{m}$ flux of a GRB at $z \gtrsim 10$ declines from $\sim 200 \mu \mathrm{Jy} 1 \mathrm{hr}$ after the burst to $\sim 1 \mu \mathrm{Jy}$ 10 days after the burst. GRBs are also attractive sources because of their intrinsic power-law spectra, from which it will be straightforward to find absorption lines. Furthermore, if GRBs occur in actively star-forming galaxies that drive winds into the surrounding IGM, the absorption spectrum can probe the host galaxy wind in detail. Because GRBs fade, they also serve as useful markers of faint galaxies at high redshifts. Subsequent deep imaging of the host could reveal details that complement the information from absorption line studies.

Observing IGM metal lines at high redshifts presents a variety of challenges. Most importantly, it requires relatively high resolution spectroscopy of faint sources in the near to midinfrared $(1 \mu \mathrm{m} \lesssim \lambda \lesssim 5 \mu \mathrm{m})$. Resolving individual winds requires a spectral resolution $R \sim c / v_{\exp } \sim 6000\left(50 \mathrm{~km} \mathrm{~s}^{-1} / v_{\text {exp }}\right)$. Such resolution is currently achievable with the NIRSPEC camera on the Keck telescope ${ }^{9}$ and with the ISAAC camera on the Very Large Telescope. ${ }^{10}$ However, any ground-based observation in this wavelength range will suffer from sky contamination that worsens significantly as the wavelength increases; thus, ground-based measurements will be limited to $z \lesssim 10$, and even then will require bright sources. The James Webb Space Telescope,${ }^{11}$ due for launch in the next decade, will have the sensitivity to probe much fainter background sources with a spectral resolution $R=5000$. The Mission Simulator ${ }^{12}$ estimates that a source with $F_{\nu} \sim 1 \mu \mathrm{Jy}$ will require a $\sim 16-24 \mathrm{hr}$ integration to achieve $S / N=10$ per resolution element at wavelengths $1.5-4 \mu \mathrm{m}$. Thus spectroscopy of high- $z$ GRB afterglows with this instrument will allow us to probe IGM metal lines at $z \gtrsim 10$ in detail.

Another challenge lies in identifying the lines. In the case of $\mathrm{C}$ IV and Si IV, the ratio of line strengths in the doublets will help to confirm the identity of the transition and hence the redshift of the line. However, the low ionization states lack doublet transitions in the relevant wavelength range. One would therefore need to identify multiple lines at each redshift in order to confirm the presence of an individual absorbing wind. The feasibility of this technique is then limited not by the strongest lines but by the weaker transitions.

Finally, we note that absorption line observations such as those we propose can be combined with imaging surveys to learn even more about the enrichment process. Adelberger et al. (2002) have already shown the usefulness of such an approach for understanding the influence Lyman-break galaxies have on their surroundings. Though difficult, similar observations over a large redshift range have the promise to map out the history of galaxy feedback on the IGM.

We thank C. Steidel and A. Shapley for illuminating discussions and J. Schaye for helpful comments that improved the manuscript. We also thank the anonymous referee for useful comments. S.R.F. thanks the Institute for Advanced Study, where much of this work was completed, for kind hospitality. AL acknowledges support from the Institute for Advanced 
Study at Princeton, the John Simon Guggenheim Memorial Fel- $\quad$ lowship, and NSF grants AST-0071019, AST-0204514.

\section{REFERENCES}

Abel, T., Bryan, G. L. \& Norman, M. L. 2002, Science, 295, 93

Adelberger, K. L., Steidel, C. S., Shapley, A. E., \& Pettini, M. 2002, ApJ, in press (astro-ph/0210314)

Aguirre, A., Hernquist, L., Katz, N., Gardner, J., \& Weinberg, D. 2001a, ApJ, 556, L11

Aguirre, A., Hernquist, L., Schaye, J., Weinberg, D. H., Katz, N., \& Gardner, J. 2001b, ApJ, 560, 599

Aguirre, A., Hernquist, L., Schaye, J., Katz, N., Weinberg, D. H., \& Gardner, J. 2001c, ApJ, 561, 521

Aguirre, A., Schaye, J., \& Theuns, T. 2002, ApJ, 576, 1

Barkana, R. \& Loeb, A. 2001, Phys. Rep., 349, 125

Bechtold, J., Crotts, A. P. S., Duncan, R. C., \& Fang, Y. 1994, ApJ, 437, L83

Becker, R. H. et al. 2001, AJ, 122, 2850

Bertschinger, E. 1985, ApJS, 58, 39

Bromm, V., Ferrara, A., Coppi, P. S., \& Larson, R. B. 2001, MNRAS, 328, 969

Bromm, V., Coppi, P. S., \& Larson, R. B. 2002, ApJ, 564, 23

Bromm, V. \& Loeb, A. 2002, ApJ, 575, 111

Cecil, G., Bland-Hawthorn, J., Veilleux, S., \& Filippenko, A. V. 2001, ApJ, 555,338

Cen, R. 2002, ApJ, submitted (astro-ph/0210473)

Ciardi, B. \& Loeb, A. 2000, ApJ, 540, 687

Cowie, L. L. \& Songaila, A. 1998, Nature, 394, 44

Cowie, L. L., Songaila, A., Kim, T., \& Hu, E. M. 1995, AJ, 109, 1522

Crotts, A. P. S., Bechtold, J., Fang, Y., \& Duncan, R. C. 1994, ApJ, 437, L79

Dekel, A. \& Woo, J. 2002, MNRAS, submitted (astro-ph/0210454)

Dove, J. B., Shull, J. M., \& Ferrara, A. 2000, ApJ, 531, 846

Ellison, S. L., Lewis, G. F., Pettini, M., Chaffee, F. H., \& Irwin, M. J. 1999, ApJ, 520, 456

Ellison, S. L., Songaila, A., Schaye, J., \& Pettini, M. 2000, AJ, 120, 1175

Fan, X., Narayanan, V. K., Strauss, M. A., White, R. L., Becker, R. H., Pentericci, L., \& Rix, H. 2002, AJ, 123, 1247

Ferland, G. J., 2001, Hazy, A Brief Introduction to Cloudy 94.00

Ferrara, A., Pettini, M., \& Shchekinov, Y. 2000, MNRAS, 319, 539

Furlanetto, S. R. \& Loeb, A. 2001, ApJ, 556, 619

Gnedin, N. Y. 1998, MNRAS, 294, 407

Gnedin, N. Y. \& Hui, L. 1998, MNRAS, 296, 44

Gunn, J. E. \& Peterson, B. A. 1965, ApJ, 142, 1633

Haiman, Z., Abel, T., \& Rees, M. J. 2000, ApJ, 534, 11

Haiman, Z., Rees, M. J., \& Loeb, A. 1997, ApJ, 484, 985

Haiman, Z. \& Loeb, A. 2001, ApJ, 552, 459

Heckman, T. M., Lehnert, M. D., Strickland, D. K., \& Armus, L. 2000, ApJS, 129,493

Heckman, T. M. \& Leitherer, C. 1997, AJ, 114, 69

Heger, A. \& Woosley, S. E. 2002, ApJ, 567, 532

Ikeuchi, S., Tomisaka, K., \& Ostriker, J. P. 1983, ApJ, 265, 583

Jenkins, A., Frenk, C. S., White, S. D. M., Colberg, J. M., Cole, S., Evrard, A. E., Couchman, H. M. P., \& Yoshida, N. 2001, MNRAS, 321, 372

Kauffmann, G. et al. 2002, MNRAS, in press (astro-ph/0205070)

Kronberg, P. P., Lesch, H., \& Hopp, U. 1999, ApJ, 511, 56

Lacey, C. \& Cole, S. 1993, MNRAS, 262, 627

Lamb, D. Q. \& Reichart, D. E. 2000, ApJ, 536, 1

Lehnert, M. D. \& Heckman, T. M. 1996, ApJ, 462, 651

Leitherer, C., Ferguson, H. C., Heckman, T. M., \& Lowenthal, J. D. 1995, ApJ, 454, L19

Leitherer, C. et al. 1999, ApJS, 123, 3
Lequeux, J., Kunth, D., Mas-Hesse, J. M., \& Sargent, W. L. W. 1995, A\&A, 301, 18

Mackey, J., Bromm, V., \& Hernquist, L. 2002, ApJ, submitted (astro$\mathrm{ph} / 0208447)$

Mac Low, M. \& Ferrara, A. 1999, ApJ, 513, 142

Madau, P., Ferrara, A., \& Rees, M. J. 2001, ApJ, 555, 92

Martin, C. L. 1998, ApJ, 506, 222

Martin, C. L. 1999, ApJ, 513, 156

Mo, H. J., Mao, S., \& White, S. D. M. 1998, MNRAS, 295, 319

Mori, M., Ferrara, A., \& Madau, P. 2002, ApJ, 571, 40

Nath, B. B. \& Trentham, N. 1997, MNRAS, 291, 505

Navarro, J., Frenk, C., \& White, S. 1997, ApJ, 490, 493

Norman, C. A., Bowen, D. V., Heckman, T., Blades, C., \& Danly, L. 1996, ApJ, 472,73

Oh, S. P. 2002, MNRAS, 336, 1021

Ostriker, J. P. \& McKee, C. 1988, Reviews of Modern Physics, 60, 1

Pettini, M., Shapley, A. E., Steidel, C. C., Cuby, J., Dickinson, M., Moorwood, A. F. M., Adelberger, K. L., \& Giavalisco, M. 2001, ApJ, 554, 981

Pettini, M., Rix, S. A., Steidel, C. C., Adelberger, K. L., Hunt, M. P., \& Shapley, A. E. 2002, ApJ, 569, 742

Phillips, A. C. 1993, AJ, 105, 486

Press, W. H. \& Schechter, P. 1974, ApJ, 187, 425

Qian, Y.-Z., Sargent, W. L. W., \& Wasserburg, G. J. 2002, ApJ, 569, L61

Qian, Y.-Z. \& Wasserburg, G. J. 2001, ApJ, 559, 925

Qian, Y.-Z. \& Wasserburg, G. J. 2002, ApJ, 567, 515

Rauch, M., Sargent, W. L. W., Barlow, T. A., \& Simcoe, R. A. 2002, ApJ, 576, 45

Ricotti, M., Gnedin, N. Y., \& Shull, J. M. 2002, ApJ, 575, 49

Salpeter, E. E. 1955, ApJ, 121, 161

Scalo, J. 1998, in ASP Conf. Series. vol. 142, The Stellar Initial Mass Function, eds. Gilmore, G. \& Howell, D. (ASP, San Francisco), 201

Scannapieco, E., Thacker, R. J., \& Davis, M. 2001, ApJ, 557, 605

Scannapieco, E., Ferrara, A., \& Madau, P. 2002, ApJ, 574, 590

Schaye, J. 2001, ApJ, 559, 507

Schneider, R., Ferrara, A., Natarajan, P., \& Omukai, K. 2002, ApJ, 571, 30

Shapley, A. E. et al. 2002, ApJ, submitted

Sheth, R. K. \& Tormen, G. 1999, MNRAS, 308, 119

Songaila, A. 2001, ApJ, 561, L153

Songaila, A. \& Cowie, L. L. 1996, AJ, 112, 335

Springel, V. \& Hernquist, L. 2002, MNRAS, submitted (astro-ph/0206393)

Steidel, C. C., Pettini, M., \& Adelberger, K. L. 2001, ApJ, 546, 665

Strickland, D. K., Heckman, T. M., Weaver, K. A., \& Dahlem, M. 2000, AJ, 120,2965

Tegmark, M., Silk, J., \& Evrard, A. 1993, ApJ, 417, 54

Theuns, T., Mo, H. J., \& Schaye, J. 2001, MNRAS, 321, 450

Theuns, T., Viel, M., Kay, S., Schaye, J., Carswell, R. F., \& Tzanavaris, P. 2002, ApJ, 578, L5

Tytler, D., Fan, X.-M., Burles, S., Cottrell, L., Davis, C., Kirkman, D., \& Zuo, L. 1995, in QSO Absorption Lines, ed. G. Meylan (Berlin: Springer)

Voit, G. M., Bryan, G. L., Balogh, M. L., \& Bower, R. G. 2002, ApJ, 576, 601

Wood, K. \& Loeb, A. 2000, ApJ, 545, 86

Woosley, S. E. \& Weaver, T. A. 1995, ApJS, 101, 181

Wyithe, J. S. B. \& Loeb, A. 2002a, Nature, 417, 923

Wyithe, J. S. B. \& Loeb, A. 2002b, ApJ, 577, 57

Wyithe, S., \& Loeb, A. 2002c, ApJ, submitted (astro-ph/0206154))

Wyithe, S., \& Loeb, A. 2002d, ApJ, in press (astro-ph/0209056) 
TABLE 1

SUPERNOVA YIELDS AND IMPORTANT TRANSITIONS

\begin{tabular}{cccccc}
\hline \hline \multirow{2}{*}{ Element } & $Y_{x}($ Scalo $)$ & $Y_{x}(\mathrm{VMS})$ & Ionization State & $\lambda_{m}(\AA)$ & $f_{\text {osc }}$ \\
\hline $\mathrm{C}$ & $0.1 \mathrm{M}_{\odot}$ & $4.1 \mathrm{M}_{\odot}$ & $\mathrm{C}$ II & 1334.5 & 0.1278 \\
& & & $\mathrm{C}$ IV & $1548.2^{\mathrm{a}}$ & 0.1908 \\
& & & $\mathrm{C}$ IV & $1550.8^{\mathrm{a}}$ & 0.09522 \\
$\mathrm{O}$ & 0.5 & 44 & $\mathrm{O}$ I & 1302.2 & 0.04887 \\
$\mathrm{Si}$ & 0.06 & \multirow{2}{*}{16} & $\mathrm{Si} \mathrm{II}$ & 1304.4 & 0.094 \\
& & & $\mathrm{Si} \mathrm{IV}$ & $1393.8^{\mathrm{a}}$ & 0.514 \\
& & \multirow{2}{*}{6.4} & $\mathrm{Si} \mathrm{IV}$ & $1402.8^{\mathrm{a}}$ & 0.2553 \\
$\mathrm{Fe}$ & 0.07 & & Fe II & 1608.5 & 0.058 \\
& & & Fe II & 2344.2 & 0.114 \\
& & & Fe II & 2382.8 & 0.300 \\
\hline
\end{tabular}

${ }^{\mathrm{a}}$ Member of doublet

TABLE 2

SUMMARY OF MODEL PARAMETERS

\begin{tabular}{ccl}
\hline \hline Symbol & Standard Value & \\
\hline$f_{*}$ & 0.1 & Definition \\
$f_{\text {esc }}$ & 0.25 & Fraction of supernova energy available for the wind \\
$f_{\text {sw }}$ & $2 f_{*}$ & Fraction of host gas entrained in outflow \\
$f_{d}$ & 0 & Fraction of swept-up Hubble flow energy available to power wind \\
$f_{m}$ & 0.1 & Fraction of swept-up material leaking into bubble interior \\
$\omega_{\mathrm{SN}}$ & $126 \mathrm{M} \odot$ & Mass of stars formed in order to produce one supernova \\
$E_{51}$ & 1 & Explosive energy per supernova, in units of $10^{51} \mathrm{erg}$ \\
$Y_{x}$ & See Table 1 & Yield of element $x$ per supernova \\
$C_{A}$ & 1 & Areal covering fraction of metals \\
$C_{m}$ & 1 & Fraction of metals contained in given ionization state \\
\hline
\end{tabular}

